\title{
Gorgonias (Octocorallia: Gorgoniidae) de las aguas someras del Pacífico Norte de Costa Rica
}

\author{
Odalisca Breedy ${ }^{1,2,3} \&$ Jorge Cortés ${ }^{1}$ \\ 1. Centro de Investigación en Ciencias del Mar y Limnología (CIMAR), Universidad de Costa Rica, San Pedro, 11501- \\ 2060 San José, Costa Rica; odaliscab@gmail.com \\ 2. Centro de Investigación en Estructuras Microscópicas (CIEMic), Universidad de Costa Rica, San Pedro, 11501-2060 \\ San José, Costa Rica \\ 3. Smithsonian Tropical Research Institute, Panamá, República de Panamá.
}

Recibido 06-VI-2014. C Corregido 17-VIII-2014. Aceptado 27-IX-2014.

\begin{abstract}
Shallow water Gorgonians (Octocorallia: Gorgoniidae) from the North Pacific of Costa Rica. Octocorals are characteristic and abundant components of shallow waters, rocky walls, and coral reef. Octocoral species richness of the Costa Rican Pacific has been addressed in several taxonomic publications. However, they have not been studied in terms of ecology, due to the problematic identification, especially in the field and the difficult access to specific localities for this type of research. In 2012 we explored some localities (less than 35 $\mathrm{m}$ deep) and evaluated the octocorallian fauna, recording species richness and occurrence. Our objective was to record octocoral species and to facilitate their identification by providing a taxonomic key for future studies We identified 10 species of Pacifigorgia, nine of Leptogorgia and four of Eugorgia. Here we include photographs of sclerites and of preserved and live octocorals. Rev. Biol. Trop. 62 (Suppl. 4): 43-62. Epub 2014 Diciembre 01.
\end{abstract}

Key words: biodiversity, identification key, Eastern Pacific, gorgonians, octocorals, taxonomy.

Los octocorales son componentes abundantes y característicos de las aguas someras sobre promontorios rocosos, arrecifes de coral y en sedimentos blandos (Hickson, 1928, Alderslade, 1984). En algunas partes contribuyen substancialmente con la formación de la estructura arrecifal (Kocurko, 1987). Estos organismos albergan una fauna muy particular, e.g. esponjas (Zea, 1993), moluscos (Gerhardt, 1990), poliquetos (Vreeland \& Lasker, 1989), crustáceos (Wirtz \& De Grave 2010) y peces (Lasker, 1985), entre otros. Además, los octocorales son notables por la abundancia en productos naturales con una importancia real o potencial en la investigación biomédica, farmacológica y terapéutica de muchas enfermedades (Bandurraga \& Fenical, 1985, Wright et al., 1989; Coll, 1992; Sammarco \& Coll, 1992; Baker \& Scheuer, 1994; Rodríguez \& Ramírez, 1994; Rodríguez et al., 1995; Gutiérrez et al.,
2006; Díaz-Marrero et al., 2009; Díaz-Marrero et al., 2011).

En general los octocorales son especies longevas, en estudios recientes en la Gran Barrera de Australia se ha encontrado que tienen niveles bajos de depredación y no muestran estacionalidad, por lo que han demostrado ser indicadores apropiados de la degradación ambiental ya que la abundancia de especies está determinada por el ambiente físico y la calidad de agua (Fabricius \& Alderslade, 2001). También han sido usados como bio-indicadores de estrés ambiental en arrecifes del Caribe (García-Parrado \& Alcolado, 1996). Además de su importancia ecológica, los octocorales están entre los organismos más espectaculares del mar por lo que son de interés económico como atracciones turísticas.

La fauna de Octocorallia en el Pacifico Oriental ha sido motivo de intensos estudios 
taxonómicos en la última década, se han revisado y verificado, los géneros más abundantes de la familia Gorgoniidae (Breedy \& Guzman, 2002, 2003a, b, 2007; Breedy et al., 2009), la más abundante en la región tropical y subtropical del Pacífico Oriental, y un género de Plexauridae (Breedy \& Guzmán, 2011) y se está trabajando en dos familias más. Se han descrito 23 especies nuevas (Breedy, 2001; Breedy \& Guzman, 2004, 2005a, b, 2008, 2012, 2013; Williams \& Breedy, 2004; Guzman \& Breedy, 2008, 2012; Breedy \& Cortés, 2011; Breedy et al., 2012; Breedy et al., 2013) provenientes de Costa Rica, Panamá, México y Ecuador.

El mayor esfuerzo de muestreo se ha concentrado en Panamá y en menor medida en algunas áreas de Costa Rica tales como Parque Nacional Isla del Coco (Breedy \& Cortés, 2008), Reserva Biológica Isla del Caño, Golfo Dulce, Puerto Jiménez, Islas Murciélago, Bahía Culebra y Bahía Salinas, sin embargo, queda mucho por explorar. A pesar que desde el punto de vista taxonómico la esta fauna ha sido bien caracterizada, desde el punto de vista ecológico no se han hecho estudios, solamente observaciones de campo que aparecen en los trabajos antes mencionados. Esto se debe en parte a la problemática que hay para identificar las especies en el campo y también al difícil acceso a los sitios donde se encuentran las poblaciones.

En 2012 como parte del proyecto Estudios científicos en el área costera del Pacífico Norte, Costa Rica para evaluar el estado de las comunidades coralinas de las aguas someras tuvimos la oportunidad de explorar algunos sitios (menos de $35 \mathrm{~m}$ de profundidad) y evaluar la fauna de Octocorallia. Se elaboró una lista de la fauna de Octocorallia por sitio visitado para los conteos de biodiversidad (Morales 2013, informe técnico, CIMAR).

El objetivo de este trabajo es informar sobre la aparición de las especies de octocorales en el área y facilitar la identificación de esta fauna por medio de una clave para futuros estudios. La clave incluye solamente los géneros de la familia Gorgoniidae ya que éstos han sido motivo de revisiones taxonómicas actualizadas (op cit.) y por lo tanto se tiene un mayor grado de certeza sobre las especies identificadas.

\section{Morfología y Sistemática de octocora-} les: Los octocorales son celenterados coloniales que se caracterizan por poseer pólipos con ocho tentáculos pinnados. Los pólipos emergen de un tejido basal común llamado cenénquime y embebidas en éste se encuentran estructuras microscópicas llamadas escleritas, que son elementos esqueléticos calcáreos que se encargan de la dureza y soporte de la colonia y de algunas partes del cuerpo del pólipo (Grasshoff, 2001). La taxonomía de los octocorales se basa en la morfología de la colonia, estructura y presencia de ejes, escleritas, y el color de la colonia y de las escleritas. La forma en que se combinan estas características es la que define las especies morfológicamente. En la subclase Octocorallia se distinguen básicamente tres órdenes: Helioporacea (coral azul), Pennatulacea (plumas de mar) y Alcyonacea (corales suaves y gorgonias).

Las gorgonias, de las familias Gorgoniidae y Plexauridae representan la fauna más abundante en las aguas someras del Pacifico de Costa Rica. Las gorgonias presentan muchas formas de crecimiento, desde colonias incrustantes hasta formas erectas arborescentes y enormes abanicos. Estas colonias se caracterizan por la presencia de un eje central escleroprotéico constituido por gorgonina y con diversos grados de mineralización interna (Bayer \& Macintyre, 2001). El eje está rodeado por una capa de cenénquime donde se encuentran las escleritas y los pólipos (Bayer, 1961).

\section{MATERIALES Y MÉTODOS}

Área de estudio: Los sitios explorados se presentan en el Cuadro 1. Se muestrearon tres sitios en cada región, escogidos de acuerdo con reportes de pescadores locales y de operadores de turismo sobre la presencia de estos animales. La inspección se llevó a cabo por medio de buceo (SCUBA) hasta $35 \mathrm{~m}$ de profundidad. Los muestreos representan un sondeo 
CUADRO 1

Sitios explorados en el Pacífico Norte de Costa Rica.

TABLE 1

North Pacific Costa Rica, explored sites.

\begin{tabular}{lc}
\multicolumn{1}{c}{ Localidad } & Abreviación \\
Bahía Salinas & BS \\
Bahía Santa Elena & BSE \\
*Islas Murciélago & IM \\
Golfo de Santa Elena & GSE \\
Bahía Culebra & BC \\
San Juanillo & SJ \\
Playa Sámara/Carrillo & SAM \\
Malpaís & MP \\
*Reserva Cabo Blanco/Cabuya & RCB \\
Golfo de Nicoya & GN \\
\hline
\end{tabular}

(*) Sitios con algún nivel de protección por parte SINAC, MINAE. preliminar de la riqueza de especies en el área, no son ni intensivos, ni representativos.

Distribución: Se registraron los sitios en que se encontraron especies de gorgonias y la profundidad, tanto para las especies obtenidas en este estudio como para las informadas en la literatura. Con base en esto se hizo el Cuadro 2 .

Metodología: Las especies se identificaron in situ o se recolectaron para su identificación posterior en el laboratorio siguiendo los procedimientos establecidos (Breedy \& Guzman, 2002). Para el estudio microscópico de las escleritas, se tomaron fragmentos pequeños de las puntas de las ramas de las

\section{CUADRO 2}

Lista de especies de gorgonias (familia Gorgoniidae) encontradas en algunos arrecifes rocosos del Pacífico Norte de Costa Rica.

TABLE 2

Gorgonian species list from some north Pacific rocky reefs in Costa Rica.

\begin{tabular}{|c|c|c|c|c|c|c|c|c|c|c|c|}
\hline Especie & $\mathrm{BS}$ & BSE & *IM & GSE & $\mathrm{BC}$ & SJ & SAM & MP & $* \mathrm{RCB}$ & GN & Profundidad (m) \\
\hline Eugorgia bradleyi & & & & & & & & & & & 8-12 \\
\hline Eugorgia daniana & & & & & & & & & & & 15 \\
\hline Eugorgia nobilis & & & & & & & & & & & $8-12$ \\
\hline Eugorgia rubens & & & & & & & & & & & 25 \\
\hline Leptogorgia alba & & & & & & & & & & & $5-25$ \\
\hline Leptogorgia cofrini & & & & & & & & & & & $10-12$ \\
\hline Leptogorgia cortesi & & & & & & & & & & & 30 \\
\hline Leptogorgia cuspidata & & & & & & & & & & & $8-12$ \\
\hline Leptogorgia diffusa & & & & & & & & & & & 20 \\
\hline Leptogorgia ignita & & & & & & & & & & & $6-8$ \\
\hline Leptogorgia pumila & & & & & & & & & & & 15 \\
\hline Leptogorgia rigida & & & & & & & & & & & $5-12$ \\
\hline Leptogorgia regis & & & & & & & & & & & $40-50$ \\
\hline Pacifigorgia adamsii & & & & & & & & & & & 14 \\
\hline Pacifigorgia cairnsi & & & & & & & & & & & 12 \\
\hline Pacifigorgia eximia & & & & & & & & & & & 20 \\
\hline Pacifigorgia firma & & & & & & & & & & & $5-10$ \\
\hline Pacifigorgia irene & & & & & & & & & & & $10-20$ \\
\hline Pacifigorgia rubicunda & & & & & & & & & & & $10-25$ \\
\hline Pacifigorgia samarensis & & & & & & & & & & & $10-12$ \\
\hline Pacifigorgia senta & & & & & & & & & & & $45-50$ \\
\hline Pacifigorgia stenobrochis & & & & & & & & & & & $15-25$ \\
\hline Pacifigorgia tupperi & & & & & & & & & & & $25-28$ \\
\hline Total de especies & 7 & 7 & 8 & 2 & 3 & 8 & 9 & 5 & 10 & 4 & \\
\hline
\end{tabular}

(*) Sitios con algún nivel de protección por parte SINAC, MINAE. 
colonias se trataron con hipoclorito de sodio (cloro casero) para disociarlas del tejido, y se observaron directamente en un microscopio invertido Olympus LX 51, con el cual se tomaron fotografías para observar el color y la abundancia relativa de las escleritas, y hacer medidas. Las fotografías de microscopio electrónico de barrido que se presentan en la figura 2 fueron obtenidas con base en la metodología propuesta por Breedy \& Guzman, (2002) y utilizando el MEB Hitachi 3700, 2360 y 570, con la idea de mostrar éstas estructuras con mayor detalle. La terminología utilizada en la clave se basa en el glosario propuesto por Calvo-Shadid \& Breedy-Shadid (2002). Se elaboró una clave para la identificación de las especies con base en las características morfológicas internas y externas de las colonias y cada especie se ilustra con fotografías de las colonias y/o de las escleritas que están depositadas en el Museo de Zoología, Universidad de Costa Rica. Cuando fue posible, se incluyen, fotografías submarinas in situ, en el área de estudio, o de otras regiones en el Pacífico Oriental (Panamá, Ecuador, Colombia) con el fin de tener una idea de las colonias vivas. Se presentan micrografías de las escleritas de cada especie para observar abundancia relativa, patrones de color y morfología. Las características utilizadas en la clave son fácilmente observables. El color se refiere a colonias preservadas secas o en etanol. La identificación se realizó con base en los trabajos Breedy y Guzman (op. cit.), Breedy et al. (2009) y Breedy et al. (2013). En los trabajos anteriores se pueden encontrar descripciones detalladas de cada especie. Se incluye en las listas y clave otras especies que se han citado en la literatura clásica (Verrill, 1868, 1868-70) aunque no se hayan encontrado en este estudio. Acrónimos usados en las figuras:

$\mathrm{MCZ}=$ Museum of Comparative Zoology

$\mathrm{PMB}=$ Parque Nacional Marino Ballena

STRI $=$ Smithsonian Tropical Research Institute $\mathrm{UCR}=$ Universidad de Costa Rica

URR $=$ Buque Urracá

USNM = United States National Museum YPM $=$ Yale Peabody Museum

\section{RESULTADOS Y DISCUSIÓN}

Los géneros presentes en el área de estudio fueron: Eugorgia con cuatro especies; Leptogorgia con nueve; y Pacifigorgia con 10 (Cuadro 2). Vale aclarar que en esta campaña se han observado seis especies de plexáuridos (géneros Heterogorgia y Muricea), pero no son evaluadas en este estudio.

Caracterización morfológica: Existe cierto grado de variabilidad intraespecífica, en cuanto al color, abundancia relativa de escleritas e incluso tamaños de escleritas por lo que los límites entre especies algunas veces son difíciles de demarcar. Por esto es importante el análisis morfológico de varios especímenes de una especie en particular y su observación, cuando sea posible, en el medio natural. Aquí se ilustran las especies y algunas variaciones morfológicas encontradas, indicadas en la clave.

\section{Familia Gorgoniidae Lamouroux, 1812 Eugorgia Verrill, 1868}

Gorgonias con ramificación flabeliformes (forma de abanico), lateral, parcialmente dicótoma, o irregularmente pinnada o subpinnada (forma de pluma), con ramas redondas o aplanadas y de un diámetro mediano. Ramificación libre, no anastomosis. Pólipos distribuidos en forma de bandas a cada lado de las ramas o distribuidos uniformemente. Pólipos se retraen completamente en el cenénquime formando montículos, ligeramente elevados, prominentes o planos. Cenénquime compuesto por husos (esclerita monoaxial recta o curveada) verrugosos, cabrestantes verrugosos y característicos cabrestantes de doble disco (con espirales de tubérculos fusionados completa o parcialmente en forma de placas). Las escleritas doble disco son las que definen el género. Escleritas antocodiales (escleritas en la base del pólipo), cuando están presentes, son husos pequeños y delgados, y/o plaquitas con bordes lobulados. Colonias y escleritas de colores brillantes, rojo, anaranjado, amarillo, morado o blanco, en general la superficie del cenénquime tiene una apariencia granulosa. 


\section{Leptogorgia Milne Edwards \& Haime, 1857}

Gorgonias con ramificación flabeliforme (forma de abanico), lateral, parcialmente dicótoma, o irregularmente pinnada o subpinnada (forma de pluma), con ramas redondas o aplanadas y de un diámetro mediano. Ramificación libre o con anastomosis ocasional (ramas entretejidas formando una malla). Pólipos distribuidos en forma de bandas a cada lado de las ramas, o distribuidos uniformemente. Pólipos se retraen completamente en el cenénquime formando montículos, ligeramente elevados, prominentes o planos. Cenénquime compuesto por husos simétricos y ornamentados, y cabrestantes (ejes radiados con dos espirales de tubérculos o verrugas, con un espacio medial definido y dos penachos terminales de protuberancias) con tubérculos que no se fusionan en discos. Escleritas antocodiales (escleritas en la base del pólipo) son bastones planos, con los extremos truncados o puntiagudos y/o plaquitas con bordes ondulados o lobulados, que pueden formar un collar en la base de los tentáculos del pólipo. Colonias y escleritas de colores brillantes, rojo, anaranjado, amarillo, morado o blanco, en general la superficie del cenénquime tiene una apariencia lisa.

\section{Pacifigorgia Bayer, 1951}

Gorgonias flabeliformes, ramificación en uno o varios planos perpendiculares o paralelos, con ramas regularmente anastomosadas formando una malla (tipo red de pesca) con diversos tamaños de ventanas (calibre de la malla), lo que caracteriza y diferencia este género de los demás. Pólipos distribuidos en forma de bandas a cada lado de las ramas, o distribuidos uniformemente. Pólipos se retraen completamente en el cenénquime formando montículos, ligeramente elevados, prominentes o planos. Cenénquime compuesto por husos simétricos y ornamentados, y cabrestantes con tubérculos que no se fusionan en discos. Escleritas antocodiales (escleritas en la base del pólipo) son bastones planos, con los extremos truncados o puntiagudos y/o plaquitas con bordes ondulados o lobulados, que pueden formar un collar en la base de los tentáculos del pólipo. Colonias y escleritas de colores brillantes, rojo, anaranjado, amarillo, morado o blanco, en general la superficie del cenénquime tiene una apariencia lisa.

Distribución: En el Cuadro 2 se muestran los sitios en que se observaron las especies de

\section{Clave preliminar para la identificación de las especies de Gorgoniidae del Pacífico norte de Costa Rica}

1a. Colonia con ramificación libre $\ldots \ldots \ldots \ldots \ldots \ldots \ldots \ldots \ldots \ldots \ldots \ldots \ldots \ldots \ldots \ldots \ldots \ldots \ldots \ldots \ldots \ldots$ (F)

1b. Colonia en forma de abanico, con las ramas entretejidas (anastomosadas) en forma de malla o red de pesca ........

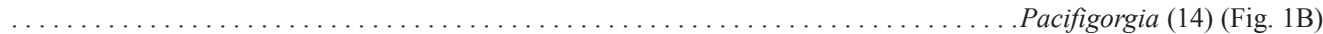

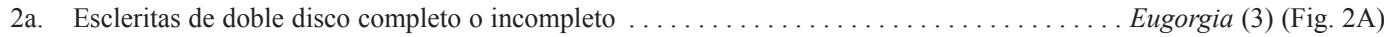

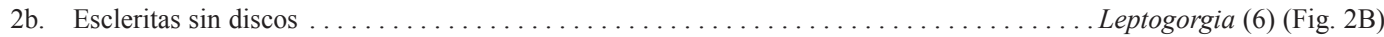

Eugorgia (2a)

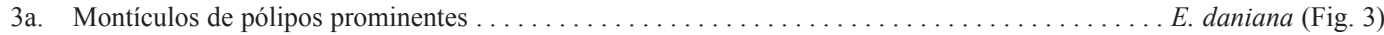

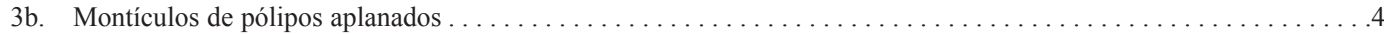

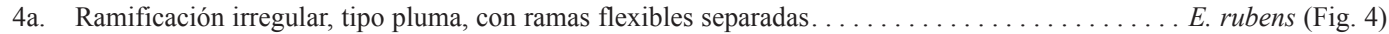

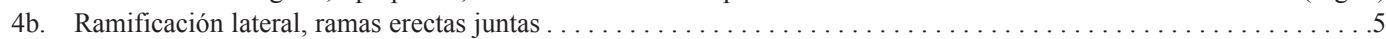

5a. Colonia amarillo intenso/anaranjada y ramas delgadas (1-2 $\mathrm{mm}$ de diámetro), todas las escleritas son

de un color anaranjado intenso $\ldots \ldots \ldots \ldots \ldots \ldots \ldots \ldots \ldots \ldots \ldots \ldots \ldots \ldots \ldots \ldots \ldots \ldots \ldots \ldots \ldots \ldots$ bradleyi (Fig. 5)

5b. Colonia rojo ladrillo y ramas gruesas (3-4 mm de diámetro), las escleritas son de un marrón rojizo,

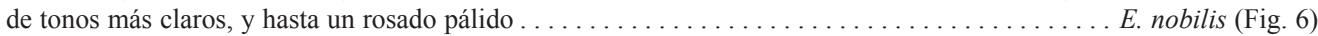

Leptogorgia (2b)

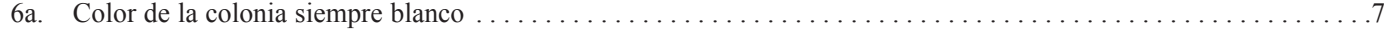

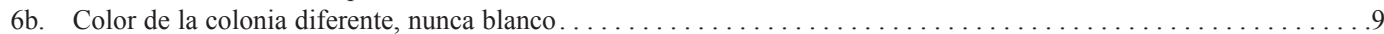


7a. Ramificación tupida, corrugada e irregular, ramas cortas curveadas, colonia no alcanza más de $8 \mathrm{~cm}$ de altura ......

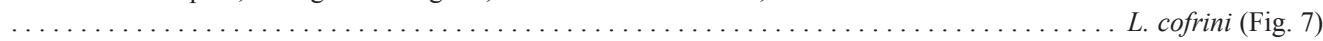

7b. Ramificación abierta, dicotómica irregular o lateral, ramas rectas y lisas, colonia alcanza hasta $60 \mathrm{~cm}$ de altura $\ldots .8$

8a. Colonia flabeliforme (forma de abanico), ramas erectas, no existe anastomosis de ramas, montículos de pólipos levemente elevados, parte distal de las ramas con contornos rectos . . . . . . . . . . . . . . . . . L. alba (Fig. 8)

8b. Colonia arbustiva, ramas flexibles, anastomosis de ramas ocasional, montículos de pólipos prominentes, parte distal

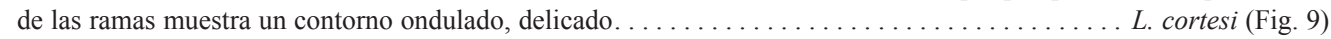

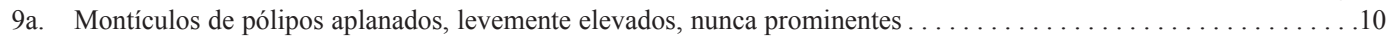

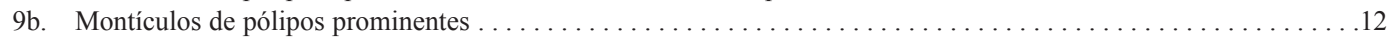

10a. Apertura monticular (hendija donde se retrae el pólipo) rodeada por un anillo distintivo amarillo o morado .......

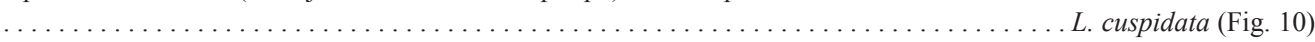

10b. Apertura monticular homogénea, no hay un anillo de color diferenciado $\ldots \ldots \ldots \ldots \ldots \ldots \ldots \ldots \ldots \ldots \ldots \ldots \ldots \ldots$

11a. Colonia anaranjado intenso, escleritas del cenénquime anaranjado brillante, escleritas antocodiales (escleritas en la

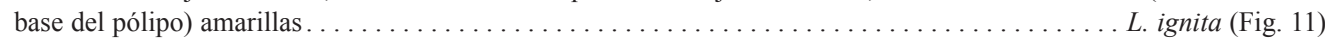

11b. Colonia morado brillante, escleritas del cenénquime rojo púrpura, escleritas antocodiales rosadas L. rigida (Fig. 12)

12a. Colonia bicolor, con las ramas terminales con el ápice de color variable (blanco/ rosado pálido/amarillo) nunca del mismo color de la base, ramas quebradizas, escleritas antocodiales blancas o amarillo pálido, miden hasta $0.12 \mathrm{~mm}$ de

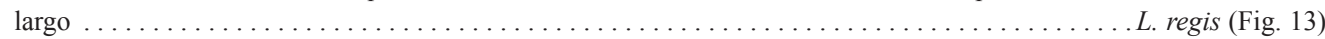

12b. Colonia nunca bicolor, de color homogéneo desde la base hasta el ápice, ramas flexibles, escleritas antocodiales nunca

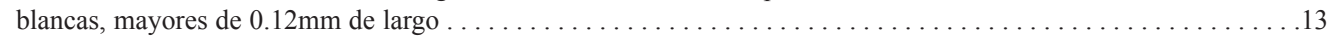

13a. Ramificación abierta, ramas flexibles, delgadas (1-2mm diámetro), montículos de los pólipos elevados 0.9-1.0mm, colonia roja, escleritas antocodiales de un anaranjado claro hasta $0.14 \mathrm{~mm}$ de largo . . . . . . . L. diffusa (Fig. 14)

13b. Ramificación tupida, ramas erectas, gruesas (2.5-3mm diámetro), montículos de los pólipos elevados $0.5-0.8 \mathrm{~mm}$, colonia rosada, escleritas antocodiales ámbar brillante hasta $0.15 \mathrm{~mm}$ de largo .

L. pumila (Fig. 15)

\section{Pacifigorgia (1a)}

14a. Malla muy cerrada con un ámbito de $1-5 \mathrm{~mm}$ de luz, con ramas entre $0.05-0.08 \mathrm{~mm}$ de diámetro, número de ventanas

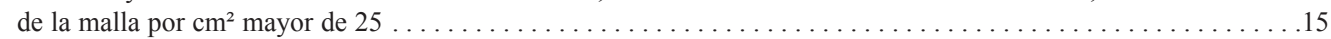

14b. Malla con un ámbito mayor de $1-5 \mathrm{~mm}$ de luz, con ramas entre $0.5-5 \mathrm{~mm}$ de diámetro, número de ventanas de la malla

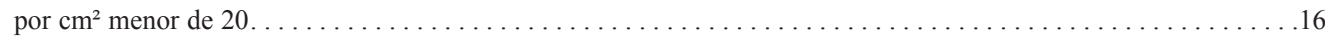

15a. Vena central gruesa, aplanada dorso ventralmente, bifurcada varias veces extendiéndose por la superficie

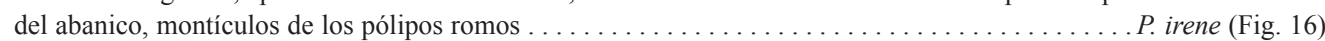

15b. Vena central generalmente ausente, cuando está presente es delgada, redondeada dorso ventralmente, y está limitada a la base de la colonia, no se bifurca, ni se extiende por la superficie del abanico,

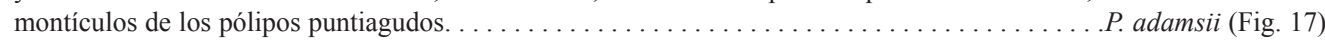

16a. Ventanas de la malla del abanico hasta $35 \mathrm{~mm}$ de lado, número de ventanas por $\mathrm{cm}^{2}$ menor de $9 \ldots \ldots \ldots \ldots 17$

16b. Ventanas de la malla del abanico hasta $12 \mathrm{~mm}$ de lado, número de ventanas por $\mathrm{cm}^{2}$ de 9 o mayor de $9 \ldots \ldots \ldots$

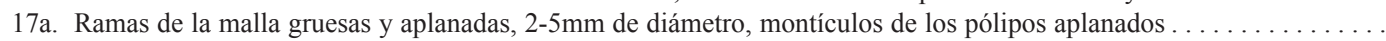
$\ldots \ldots \ldots \ldots \ldots \ldots \ldots \ldots \ldots \ldots \ldots \ldots \ldots \ldots \ldots \ldots \ldots \ldots \ldots \ldots \ldots \ldots \ldots \ldots \ldots \ldots \ldots \ldots \ldots \ldots \ldots \ldots$ stenobrochis (Fig. 18)

17b. Ramas de la malla delgadas y redondeadas, $1-1.5 \mathrm{~mm}$ de diámetro, montículos de los pólipos prominentes $\ldots \ldots 18$

18a. Colonia rosada o blancuzca, ventanas de la malla irregulares, escleritas del cenénquime

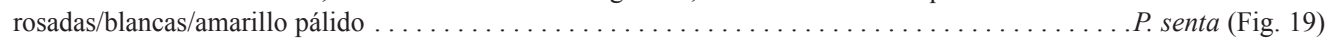

18b. Colonia rojo vivo, ventanas de la malla mayormente rectangulares, escleritas del cenénquime todas rojas .........

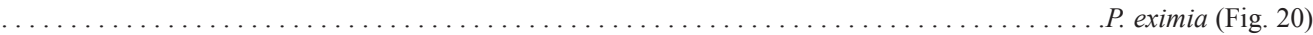

19a. Colonia morado oscuro y todas las escleritas del cenénquime rojo oscuro . . . . . . . . . . . P. tupperi (Fig. 21)

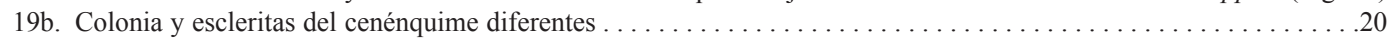

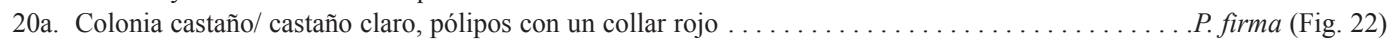

20b. Colonia en tonos de rojo/anaranjado rojizo, nunca castaño, pólipos sin collar rojo $\ldots \ldots \ldots \ldots \ldots \ldots \ldots \ldots$

21a. Colonia rojo oscuro, ventanas de la malla del abanico hasta $9 \mathrm{~mm}$ de lado, número de ventanas por $\mathrm{cm}^{2}$ hasta 9 , escleritas del cenénquime rojo intenso con un halo amarillo pálido alrededor . . . . . . . . . . P. cairnsi (Fig. 23)

21b. Colonia en tonos de anaranjado rojizo, ventanas de la malla del abanico hasta $7 \mathrm{~mm}$ de lado, número de ventanas por $\mathrm{cm}^{2}$ mayor de 9 , escleritas del cenénquime anaranjadas, amarillas o anaranjado rojizo,

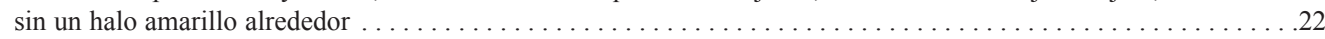

22a. Escleritas del cenénquime anaranjadas, amarillo pálido y bicolores (parte de la esclerita con uno de los colores y parte con el otro), escleritas antocodiales amarillo pálido/transparentes, hasta $0.14 \mathrm{~mm}$ de largo . . . . P. rubicunda (Fig. 24)

22b. Escleritas del cenénquime rojas, amarillas y bicolores, escleritas antocodiales amarillo oscuro,

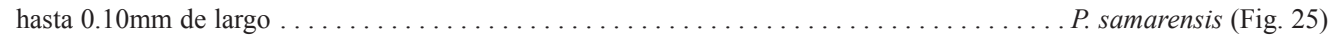


gorgonias. Leptogorgia alba y P. irene son las que aparecen en mayor cantidad de localidades, la primera en las 10 y la segunda en 6 , seguidas por P. firma ( en 5) y P. rubicunda (en 4). En cuanto a los sitios, Reserva Cabo Blanco/ Cabuya (RCB) presenta el mayor número de especies, 10, seguido por Playa Sámara/ Carrillo (SAM) con 9. El menor número de especies fue en Golfo de Santa Elena (GSE) con dos. Solamente en Islas Murciélago (IM) se encuentró una especie endémica ( $P$. tupperi). Las especies mencionadas corresponden a un ámbito batimétrico de $0-40 \mathrm{~m}$, Pacifigorgia senta y Leptogorgia regis fueron obtenidas por redes de pesca a profundidad mayor (40-50m), en bajos de las bahías de Santa Elena y Salinas o encontrados en la playa de Manzanillo y Cuajiniquil, donde los pescadores desembarcan.

\section{Observaciones de campo}

No se hizo estudios cuantitativos de abundancia, pero se observó que con respecto a Eugorgia, las colonias son muy escasas y aparecen muy dispersas sin formar agregaciones. Las de Leptogorgia forman agregaciones solamente entre $5-7 \mathrm{~m}$ de profundidad, específicamente L. cuspidata, en Bahía Salinas y L. rigida en las Islas Murciélago. Leptogorgia alba está presente en todos los sitios y se distribuye desde 5 hasta $25 \mathrm{~m}$ de profundidad y en algunos sitios es la especie dominante sin llegar a formar agregaciones tupidas como las dos especies anteriores. Pacifigorgia irene, se encuentra entre $10-20 \mathrm{~m}$ de profundidad, forman agregaciones no muy densas. Pacifigorgia firma forma pequeños grupos a 5-8m de profundidad en Bahía Culebra, Bahía Santa Elena y Playa Sámara/Carrillo, en los demás sitios, al igual que las demás especies de Pacifigorgia están representadas por una sola colonia o muy pocas.

Las poblaciones de octocorales están determinadas por factores locales como son las corriente, el oleaje, conectividad entre sitios, y especialmente la disponibilidad de substrato ya que compiten con algas, esponjas, corales, y sedimentos (Jordán-Dahlgren, 2002). Los daños de tipo mecánico como abrasión natural con el substrato u otros organismos, así como los daños no naturales como algunos artes de pesca, son las principales causas de mortalidad en octocorales, ya sea por desprendimiento o por debilitamiento de colonias que al estar dañadas son colonizadas por otros organismos que terminan quebrándolas. Algunas de las poblaciones de octocorales se encuentran en áreas impactadas por la pesca. Se observaron algunas líneas de pesca enredadas entre las rocas, anzuelos y fragmentos de red. No hay indicios de daños mecánicos en el fondo. Sin embargo, es interesante notar que las especies de aguas profundas, de más de $40 \mathrm{~m}$, como son L. regis y $P$. senta, han sido únicamente recolectadas incidentalmente con redes o líneas de pesca en el Golfo de Santa Elena. Esas especies vienen enredadas en esos artes de pesca y en muchos casos las colonias son arrancadas enteras. Esto indica que se está infringiendo un daño constante a estas poblaciones, que son diferentes de las someras, en los bajos profundos de pesca, lo cual necesita ser investigado y valorado.

\section{AGRADECIMIENTOS}

Nuestro agradecimiento a los revisores anónimos por sus comentarios. A Alexander Rodríguez por la confección de las figuras. Agradecemos al SINAC por el permiso para trabajar en las áreas protegidas, a María Marta Chavarría (Área de Conservación de Guanacaste) y Minor Lara (operador turístico) por su apoyo en la logística y el trabajo de campo y a los fotógrafos que permitieron usar sus fotografías submarinas en este trabajo. Nuestro agradecimiento a la Agencia Alemana de Cooperación GiZ a través del Proyecto BIOMARC por el apoyo económico y a la Asociación Costa Rica por Siempre. También a la Vicerrectoría de Investigación de la Universidad de Costa Rica a través del proyecto 808-B2-400. 

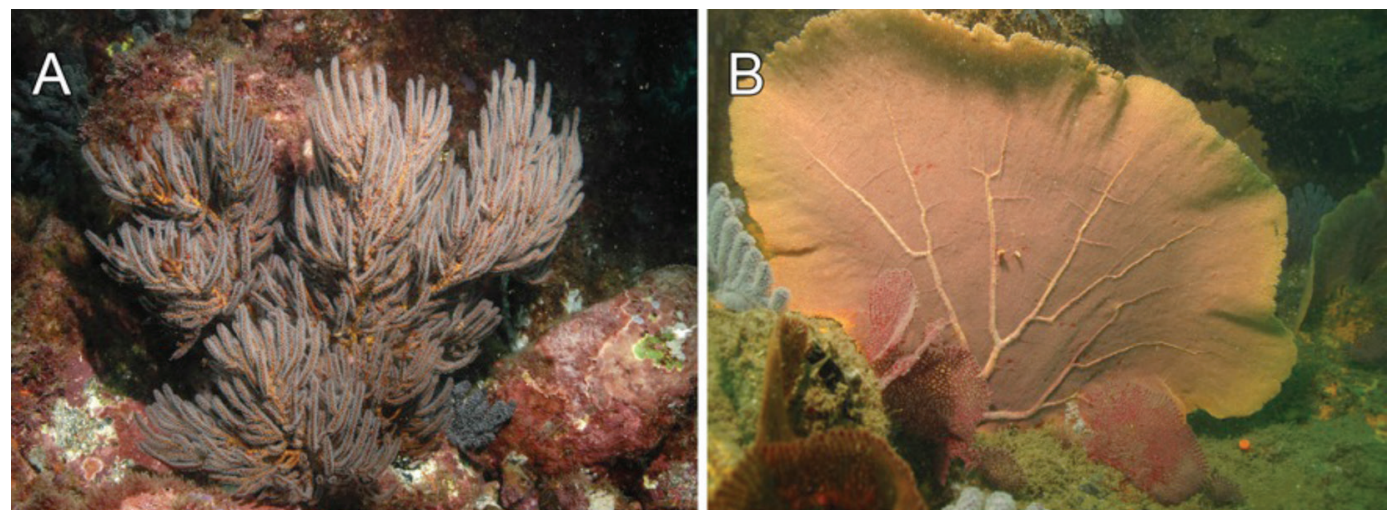

Fig. 1. (A) Colonias de Leptogorgia (L. cuspidata), en forma de ramificación libre. (B). Colonias de Pacifigorgia (P. irene) en forma de malla.

Fig. 1. (A) Leptogorgia (L. cuspidata), branched colony. (B) Pacifigorgia (P. irene), reticulate colony (net-like branching).
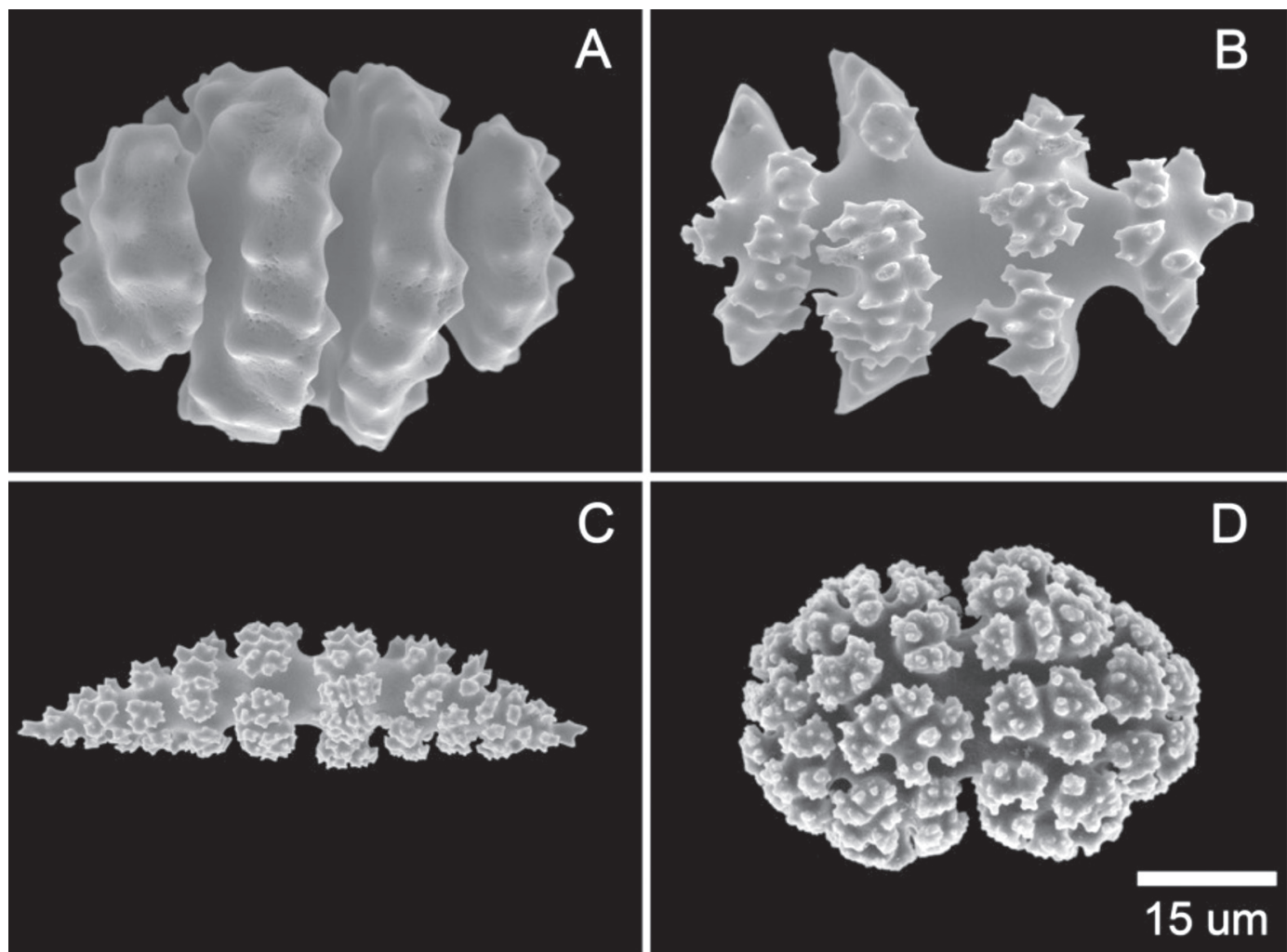

Fig. 2. Micrografía de escleritas en MEB. Escleritas de doble disco (A) Completo y (B) Incompleto. Escleritas sin discos (C) Huso. (D) Cabrestante.

Fig. 2. SEM micrographs. Double disc sclerites (A) Complete disc and (B) Incomplete disc. Sclerites without discs (C) Spindle. (D) Capstan. 


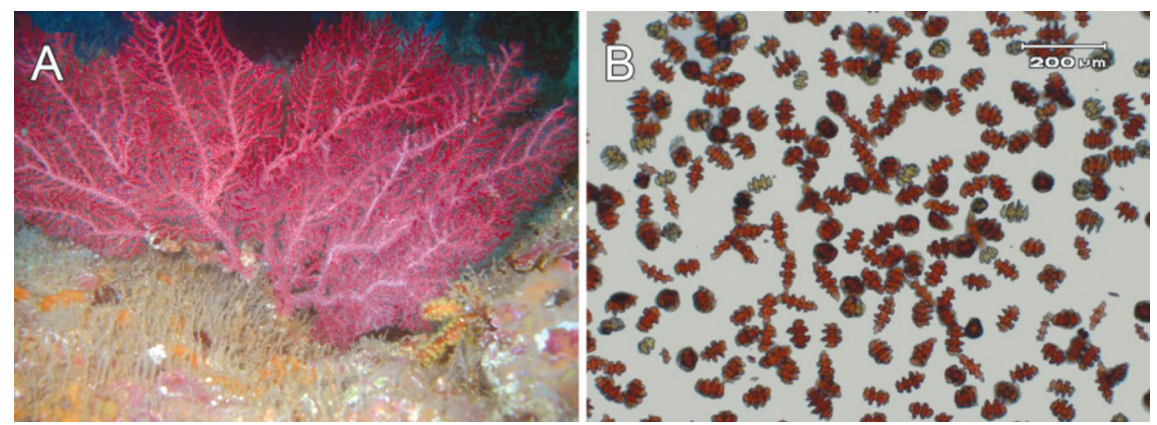

Fig. 3. Eugorgia daniana. (A) Colonia in situ, Isla de Coiba, Panamá. (B) Micrografía de escleritas.

Fig. 3. Eugorgia daniana. (A) In situ colony, Coiba Island, Panama. (B) Sclerites micrograph.
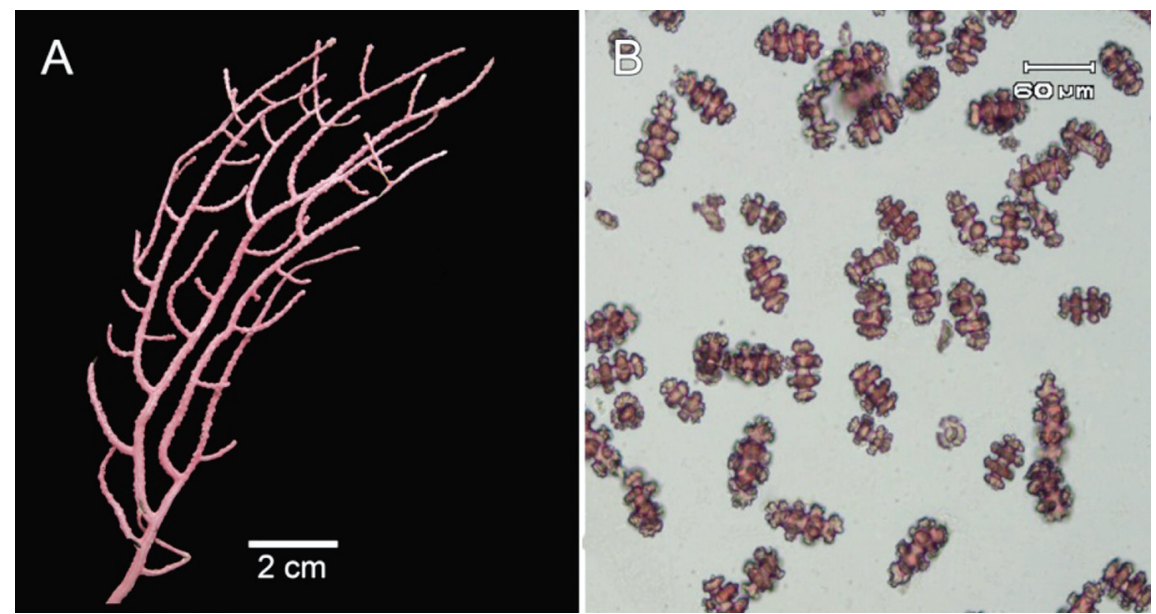

Fig. 4. Eugorgia rubens. (A) Colonia preservada, California. (B) Micrografía de escleritas.

Fig. 4. Eugorgia rubens. (A) Preserved colony, California. (B) Sclerites micrograph.
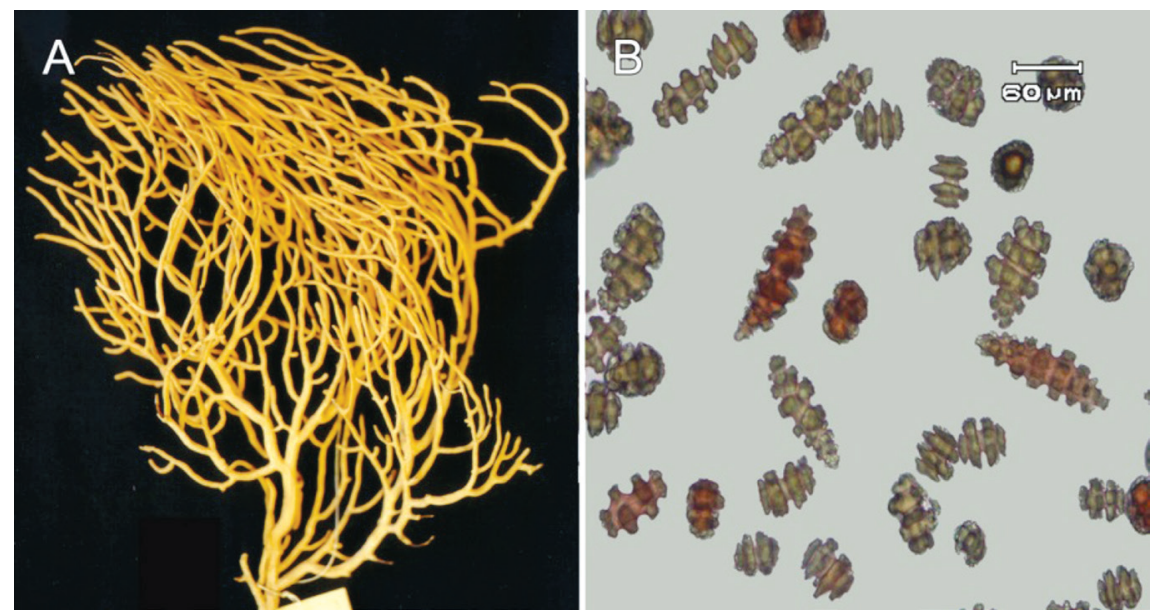

Fig. 5. Eugorgia bradleyi. (A) Colonia preservada, MCZ 7006A, Golfo de Nicoya, Costa Rica. (B) Micrografía de escleritas, MCZ 7006A.

Fig. 5. Eugorgia bradleyi. (A) Preserved colony, MCZ 7006A, Nicoya Gulf, Costa Rica. (B) Sclerites micrograph, MCZ 7006A. 

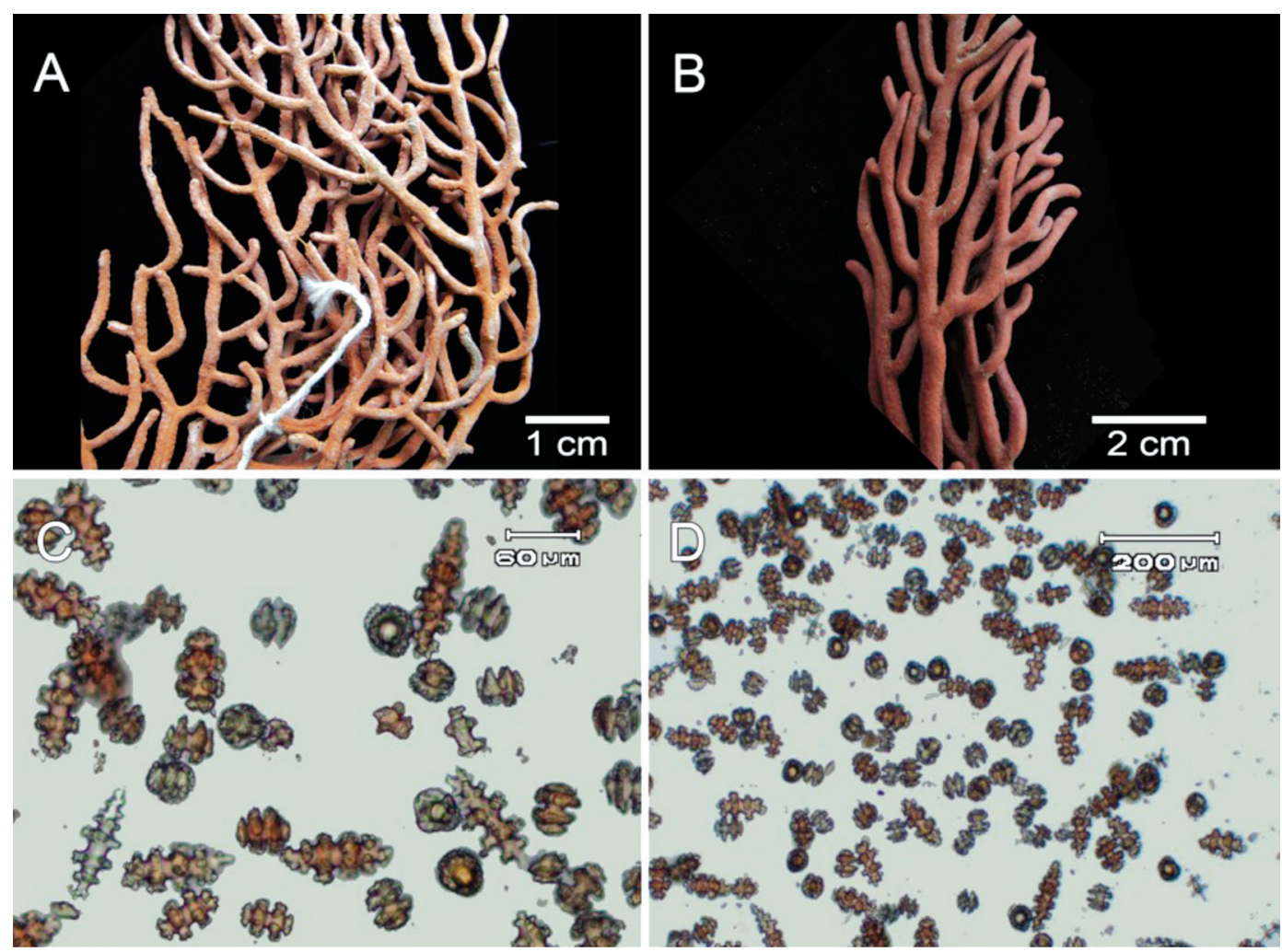

Fig. 6. Eugorgia nobilis. Colonias preservadas, morfotipos (A) USNM 50154, Paita, Perú. (B) USNM 44207, Golfo de Nicoya, Costa Rica. (C) Micrografías de escleritas, YPM 1552a.

Fig. 6. Eugorgia nobilis. Preserved colonies, morphotypes (A) USNM 50154, Paita, Perú. (B) USNM 44207, Gulf of Nicoya, Costa Rica. (C) Sclerite micrographs, YPM 1552a.

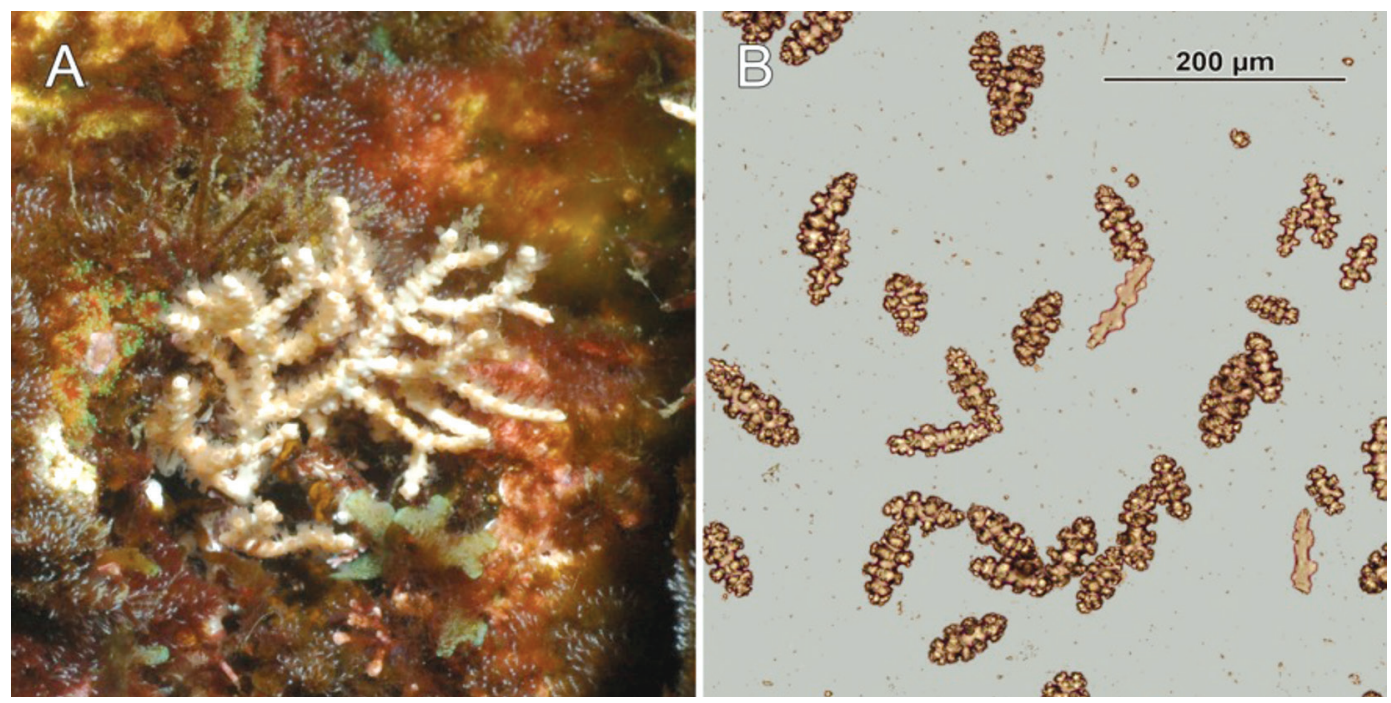

Fig. 7. Leptogorgia cofrini. (A) Colonia in situ, Isla de Coiba, fotografía G. Edgar. (B) Micrografía de escleritas. Fig. 7. Leptogorgia cofrini. (A) In situ colony, Coiba Island, photograph G. Edgar. (B) Sclerites micrograph. 

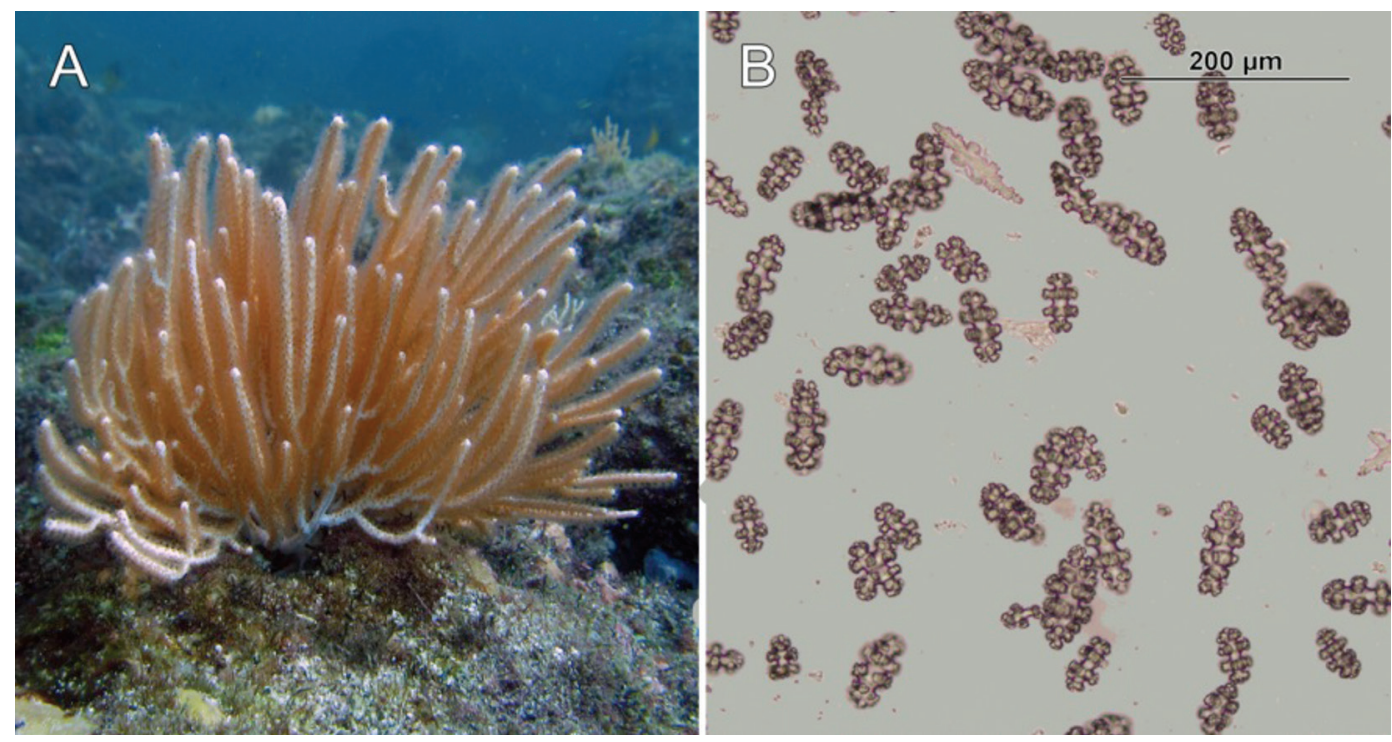

Fig. 8. Leptogorgia alba. (A) Colonia in situ, Cabo Blanco, Costa Rica, fotografía J. Nivia. (B) Micrografía de escleritas, STRI 129.

Fig. 8. Leptogorgia alba. (A) In situ colony, Cabo Blanco, Costa Rica, photograph J. Nivia. (B) Sclerites micrograph, STRI 129.
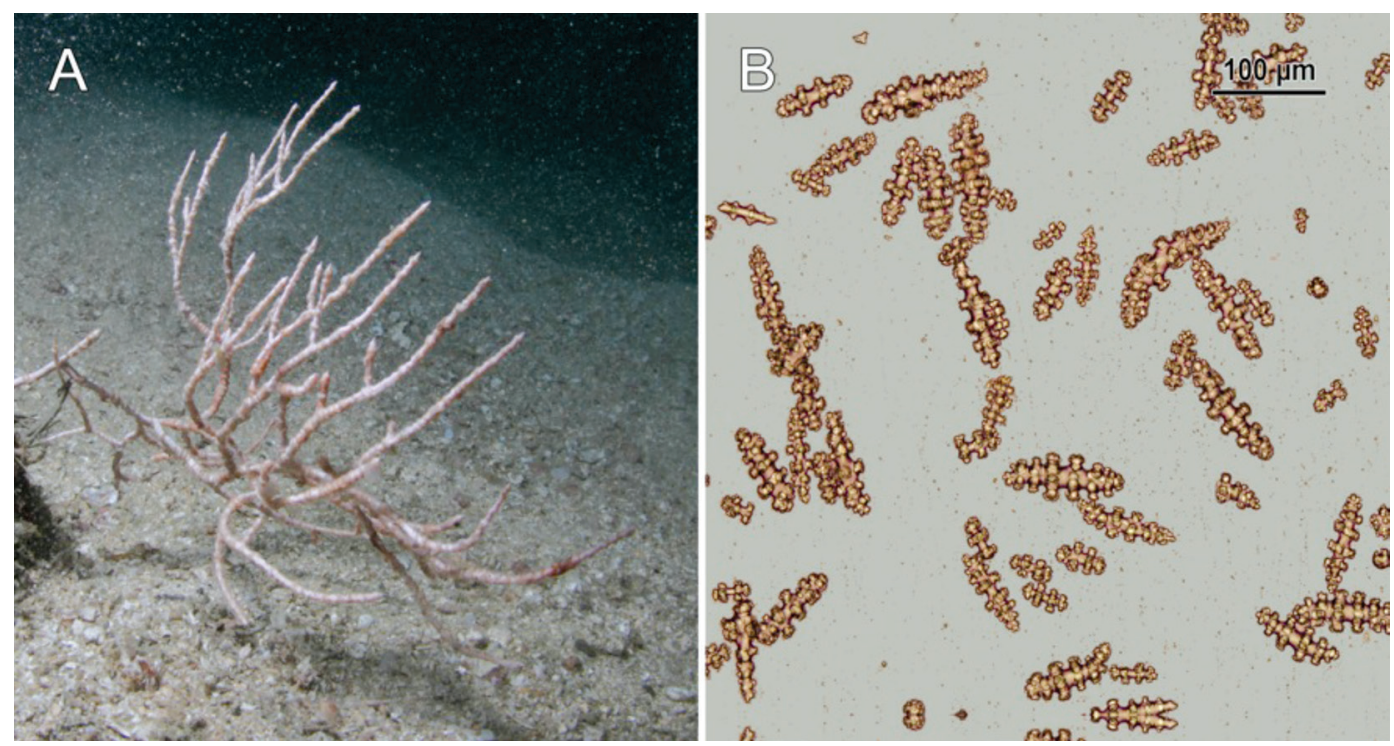

Fig. 9. Leptogorgia cortesi. (A) Colonia in situ, Golfo Dulce, Costa Rica, fotografía A. Klapfer. (B) Micrografía de escleritas. Fig. 9. Leptogorgia cortesi. (A) In situ colony, Golfo Dulce, Costa Rica, photograph A. Klapfer. (B) Sclerites micrograph. 


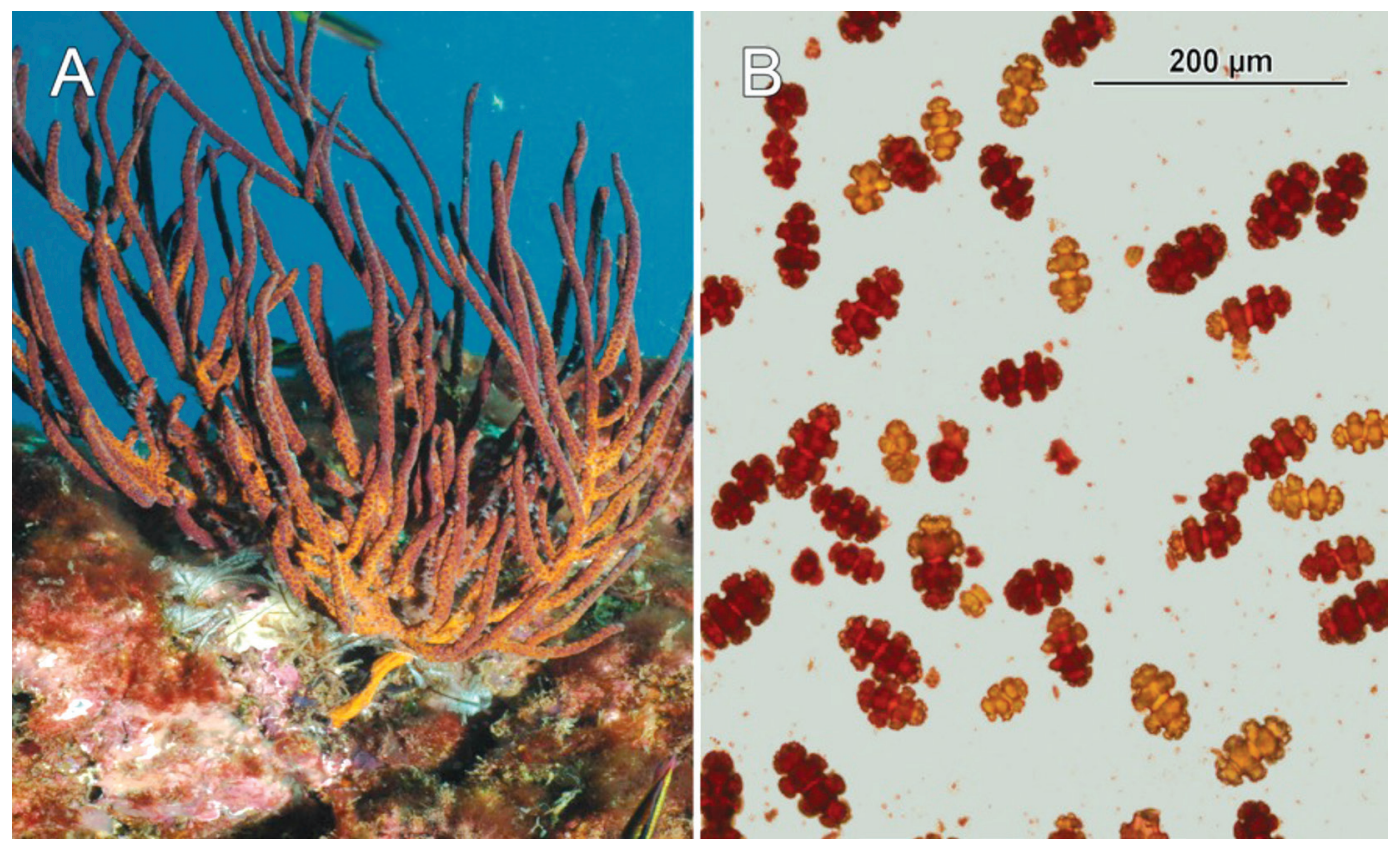

Fig. 10. Leptogorgia cuspidata. (A) Colonia in situ, Isla Coiba, Panamá, fotografía G. Edgar (B) Micrografía de escleritas. Fig. 10. Leptogorgia cuspidata. (A) In situ colony, Isla Coiba, Panama, photograph G. Edgar. (B) Sclerites micrograph.
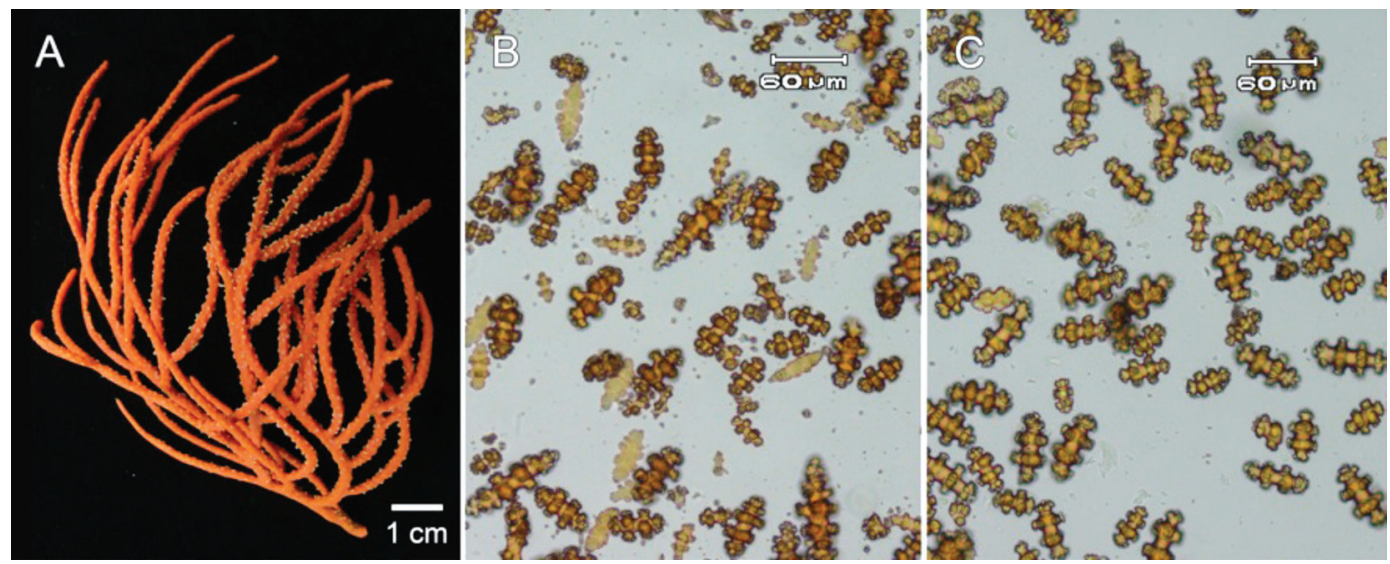

Fig. 11. Leptogorgia ignita. (A) Colonia preservada, UCR 629, Sámara, Costa Rica. (B) Micrografía de escleritas, variabilidad interespecífica, UCR 629. (C) Micrografía de escleritas, variabilidad interespecífica, UCR 514.

Fig. 11. Leptogorgia ignita. (A) Preserved colony, UCR 629 Sámara, Costa Rica. (B) Sclerites micrograph, interspecific variation, UCR 629. (C) Sclerites micrograph, interspecific variation, UCR 514. 


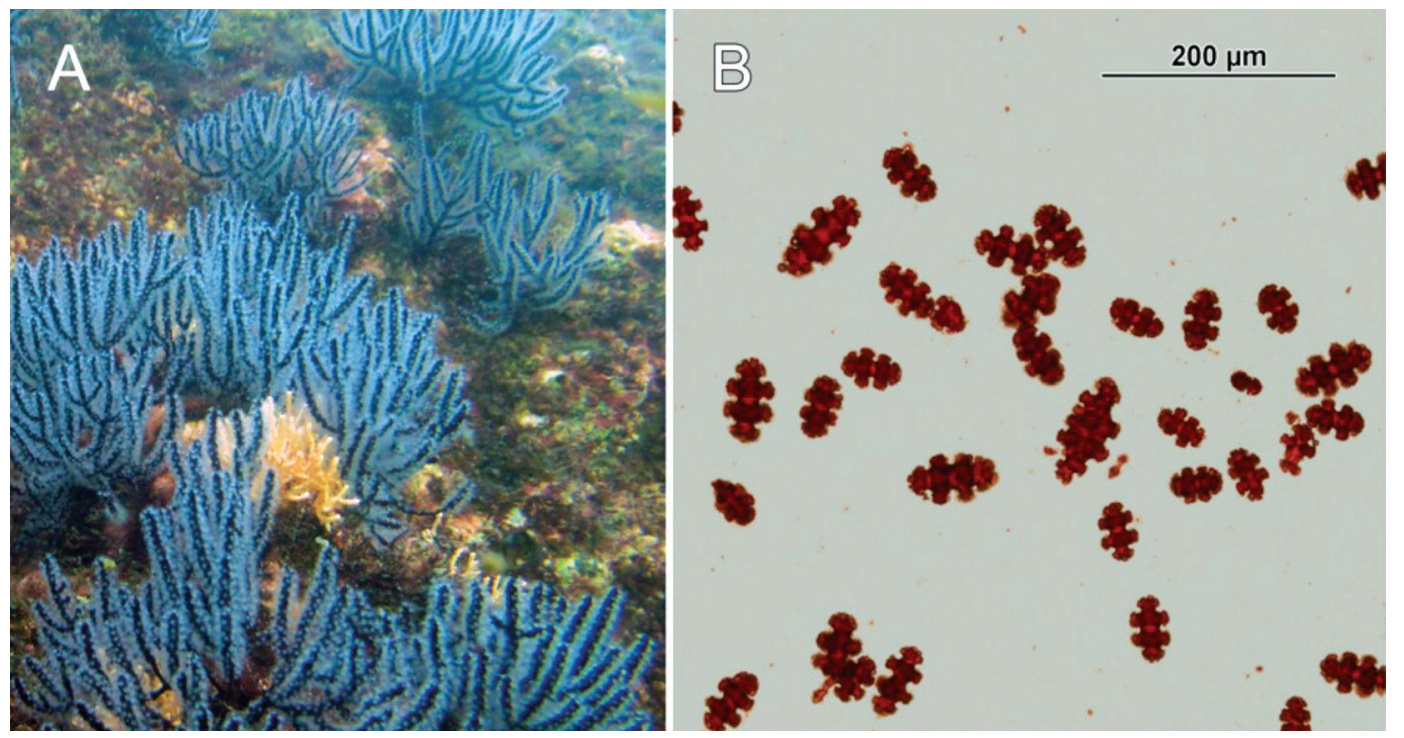

Fig. 12. Leptogorgia rigida. (A) Colonia in situ, Cabo Blanco, Costa Rica, fotografía J. Nivia. (B) Micrografía de escleritas. Fig. 12. Leptogorgia rigida. (A) In situ colony, Cabo Blanco, Costa Rica, photograph J. Nivia. (B) Sclerites micrograph.
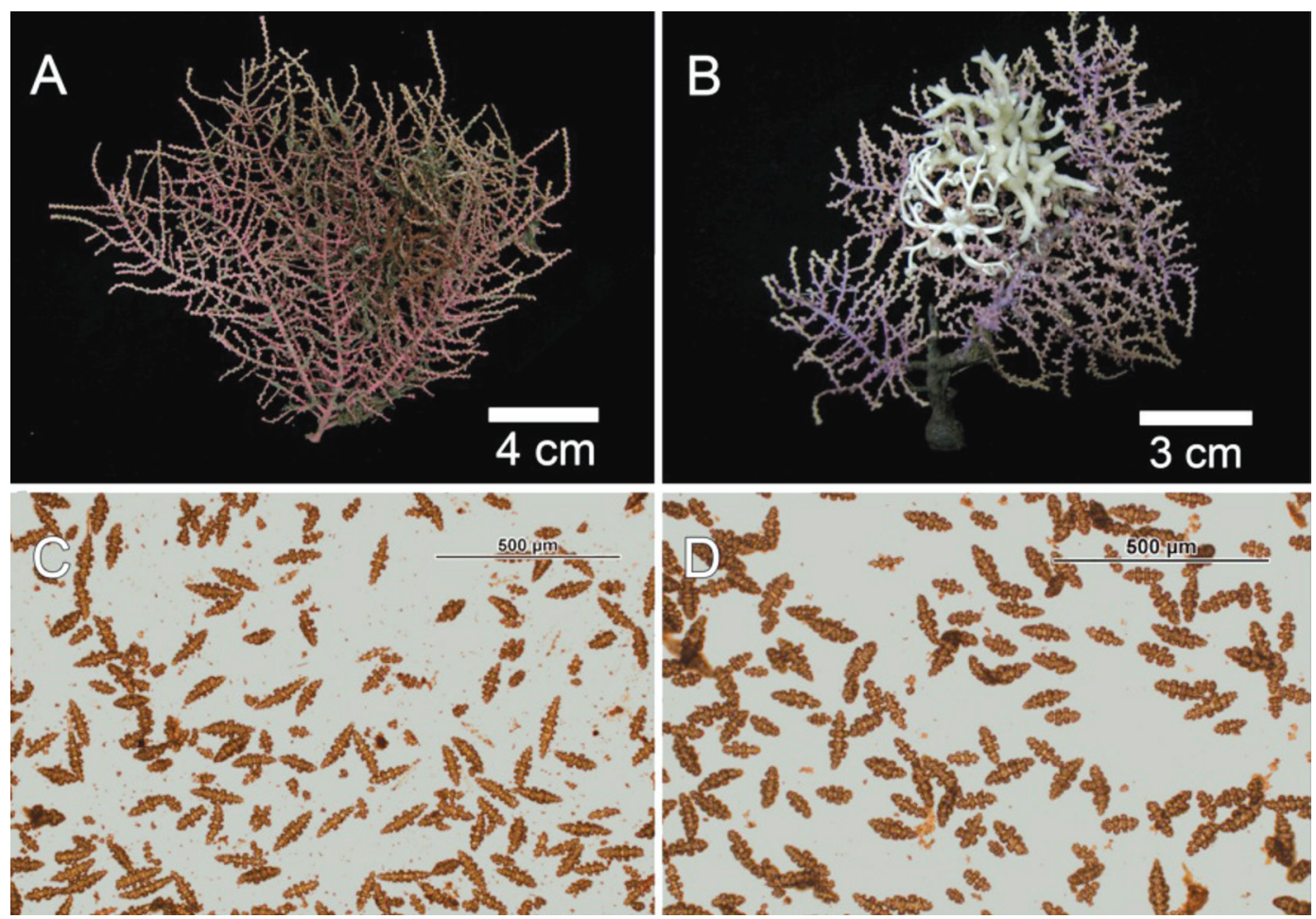

Fig. 13. Leptogorgia regis. (A) Colonia preservada, Bahía Santa Elena, Costa Rica. (B) Colonia preservada, Bahía Santa Elena, variabilidad en color. (C) Micrografía de escleritas, variabilidad interespecífica. (D) Micrografía de escleritas, variabilidad interespecífica.

Fig. 13. Leptogorgia regis. (A) Preserved colony Bahía Santa Elena, Costa Rica. (B) Preserved colony Bahía Santa Elena, interspecific variability. (C) Sclerites micrograph, interspecific variability. (D) Sclerites micrograph, interspecific variability. 

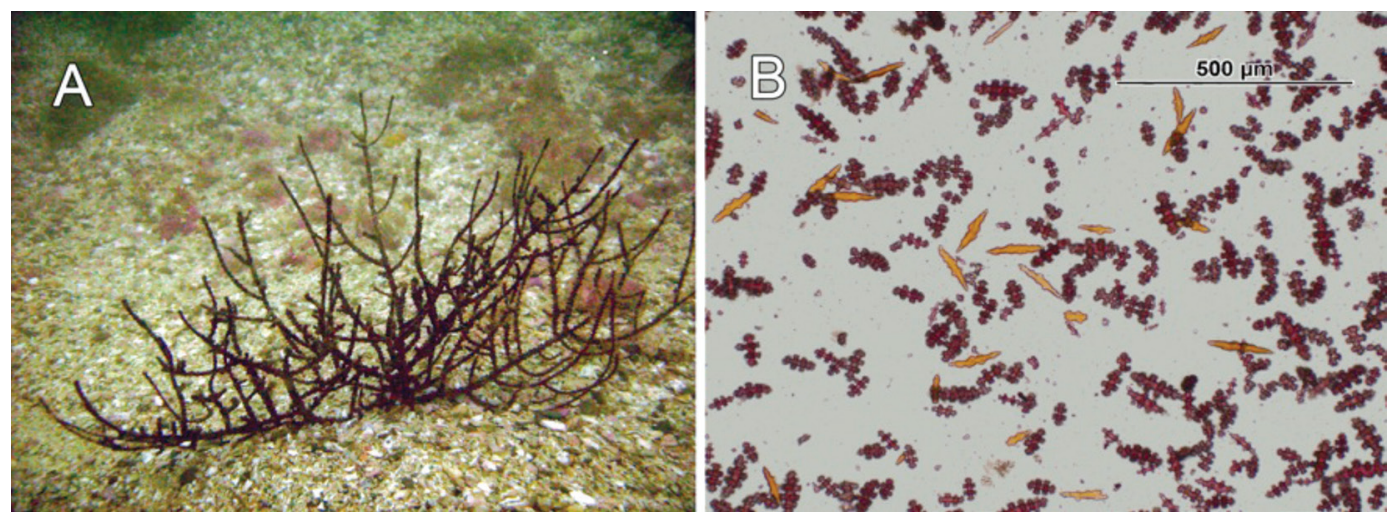

Fig. 14. Leptogorgia diffusa. (A) Colonia in situ, Parque Nacional Marino Ballena, Costa Rica. (B) Micrografía de escleritas.

Fig. 14. Leptogorgia diffusa. (A) In situ colony, Parque Nacional Marino Ballena, Costa Rica. (B) Sclerites micrograph.
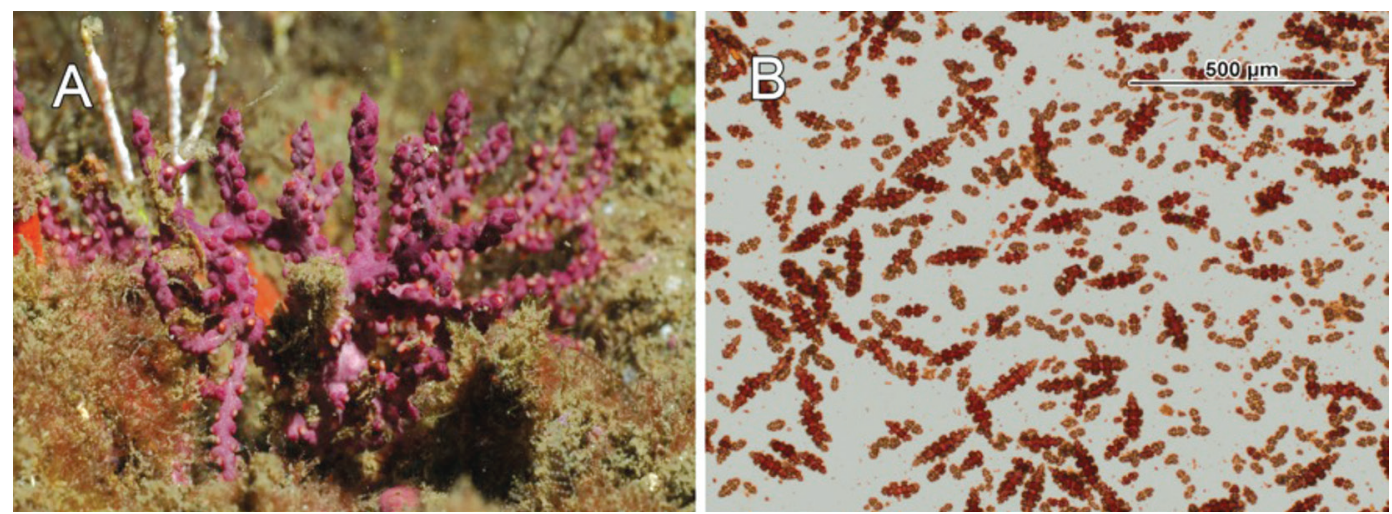

Fig. 15. Leptogorgia pumila. (A) Colonia in situ, Isla de Coiba, Panamá. (B) Micrografía de escleritas.

Fig. 15. Leptogorgia pumila. (A) In situ colony, Isla de Coiba, Panamá. (B) Sclerites micrograph.
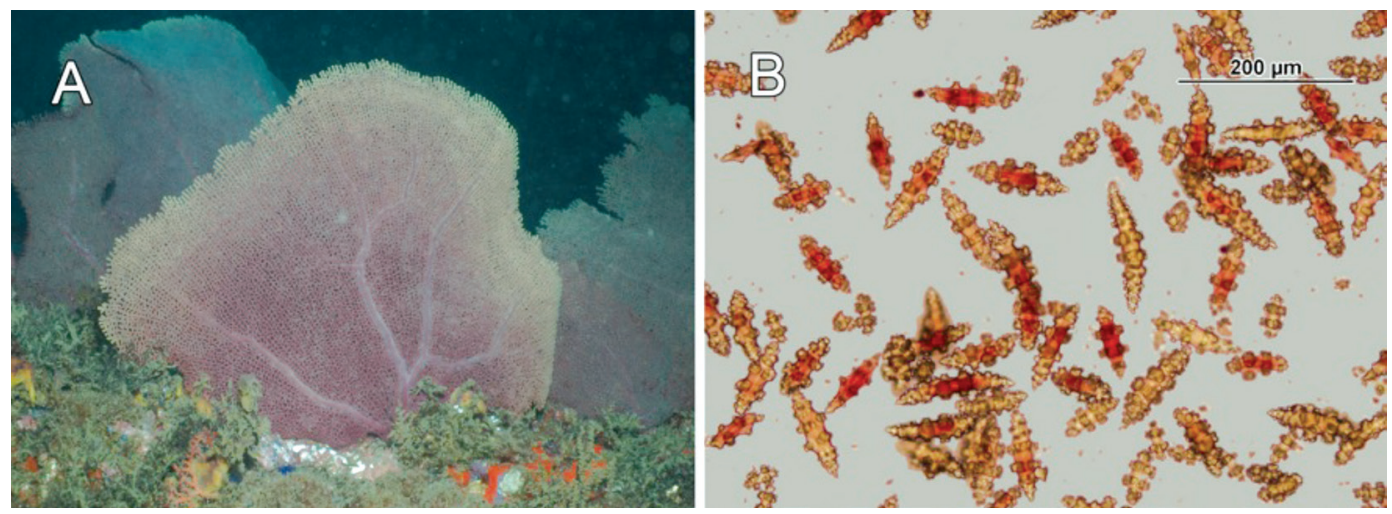

Fig. 16. Pacifigorgia irene. (A) Colonia in situ, Isla de Coiba, Panamá. (B) Micrografía de escleritas.

Fig. 16. Pacifigorgia irene. (A) In situ colony, Isla de Coiba, Panamá. (B) Sclerites micrograph. 


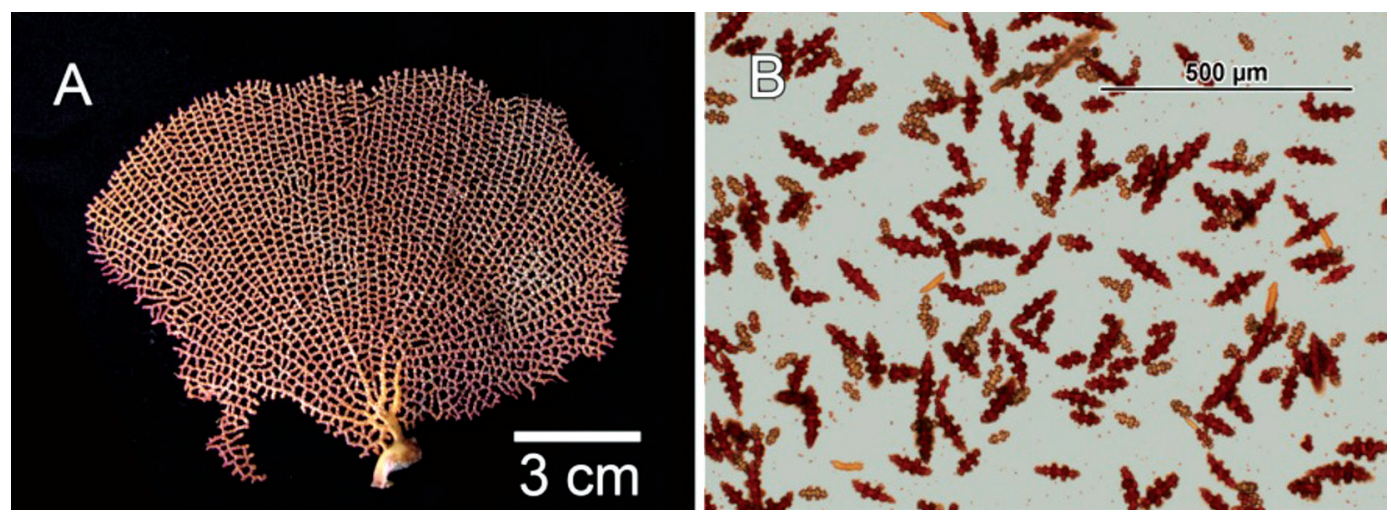

Fig. 17. Pacifigorgia adamsii. (A) Colonia preservada, URR 27-35. (B) Micrografía de escleritas, PMB-UCR. Fig. 17. Pacifigorgia adamsii. (A) Preserved colony, URR 27-35. (B) Sclerites micrograph, PMB-UCR.
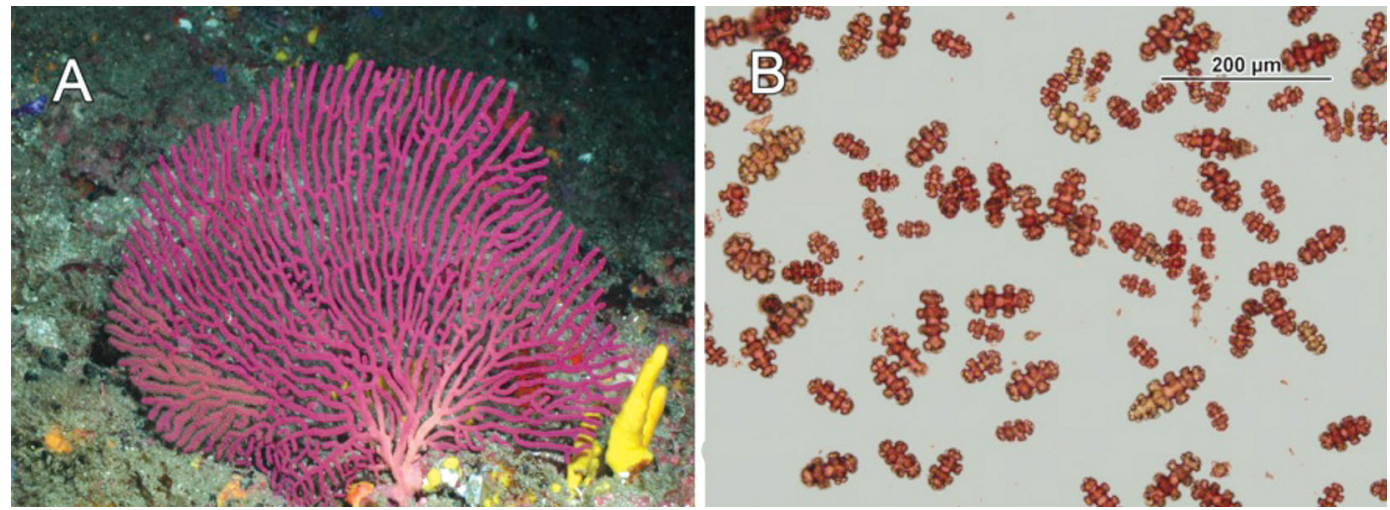

Fig. 18. Pacifigorgia stenobrochis. (A) Colonia in situ, Isla Coiba, Panamá, fotografía G. Edgar. (B) Micrografía de escleritas.

Fig. 18. Pacifigorgia stenobrochis. (A) In situ colony, Isla Coiba, Panamá, photograph G. Edgar. (B) Sclerites micrograph.
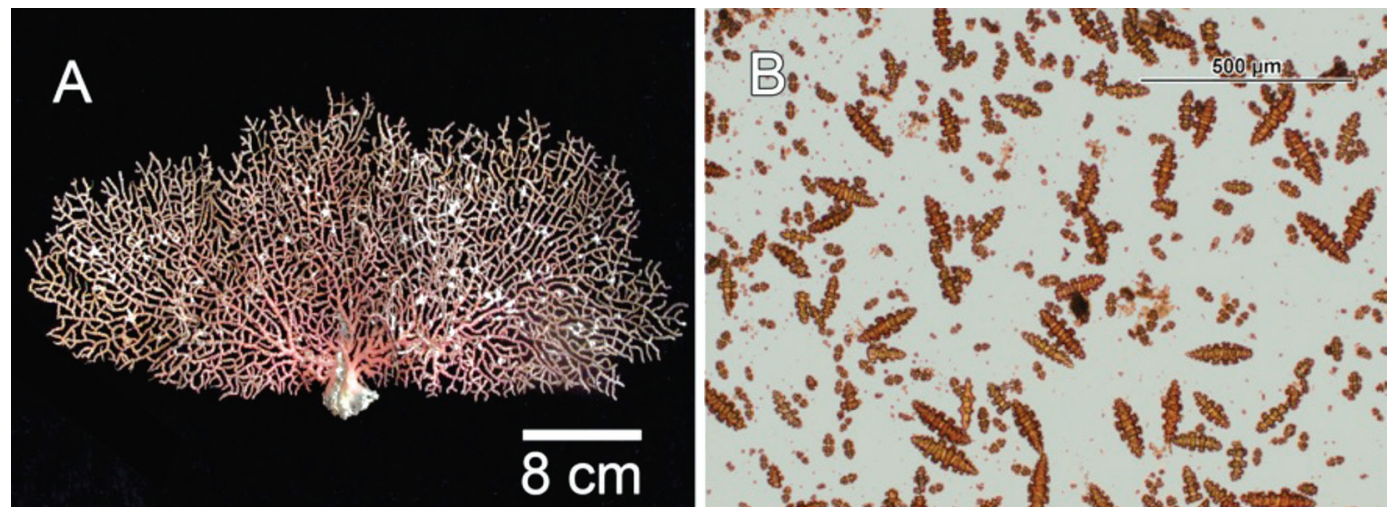

Fig. 19. Pacifigorgia senta. (A) Colonia preservada, UCR 2309, Bahía Salinas, Costa Rica. (B) Micrografía de escleritas.

Fig. 19. Pacifigorgia senta. (A) Preserved colony, UCR 2309, Bahía Salinas, Costa Rica. (B) Sclerites micrograph. 


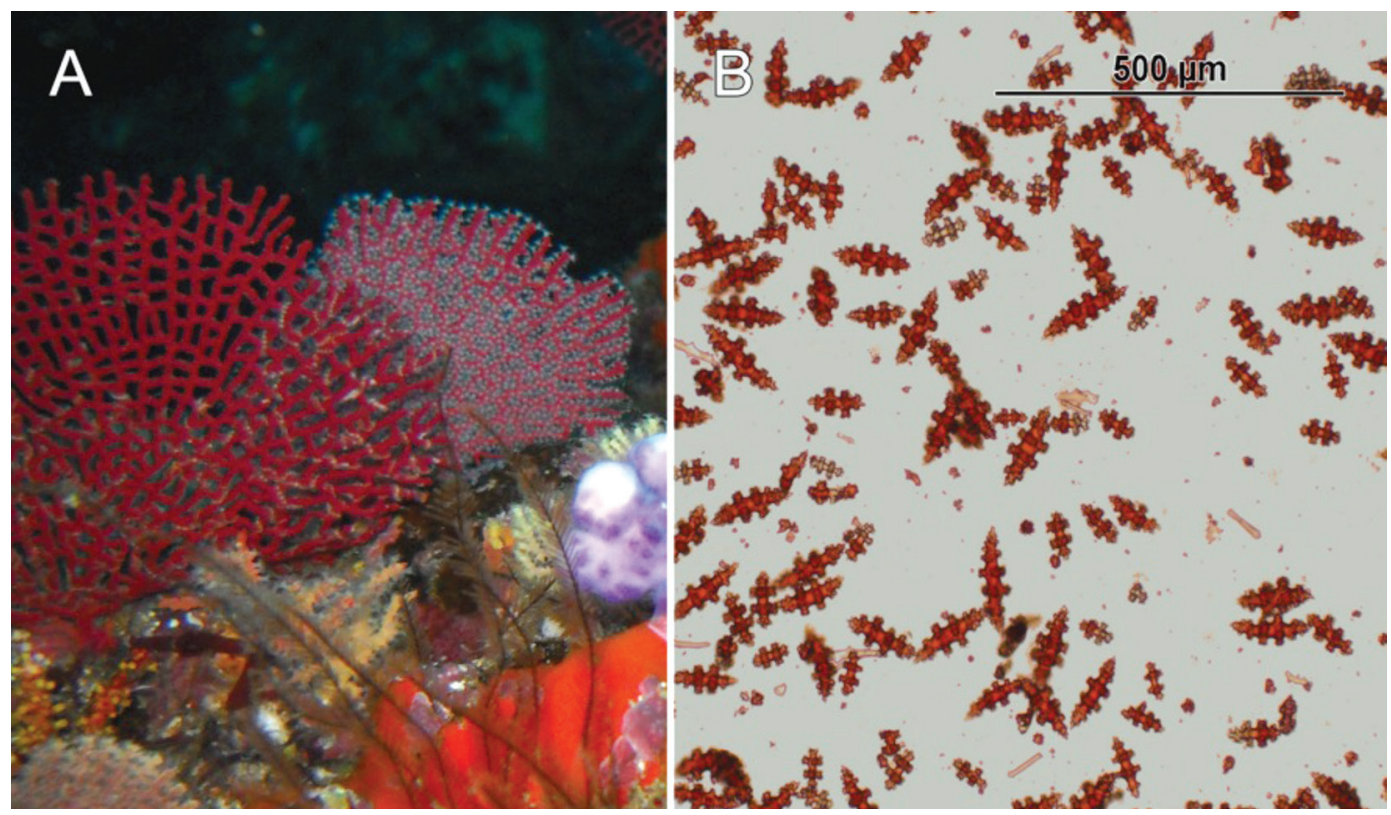

Fig. 20. Pacifigorgia eximia. (A) Colonia in situ. (B) Micrografía de escleritas.

Fig. 20. Pacifigorgia eximia. (A) In situ colony. (B) Sclerites micrograph.
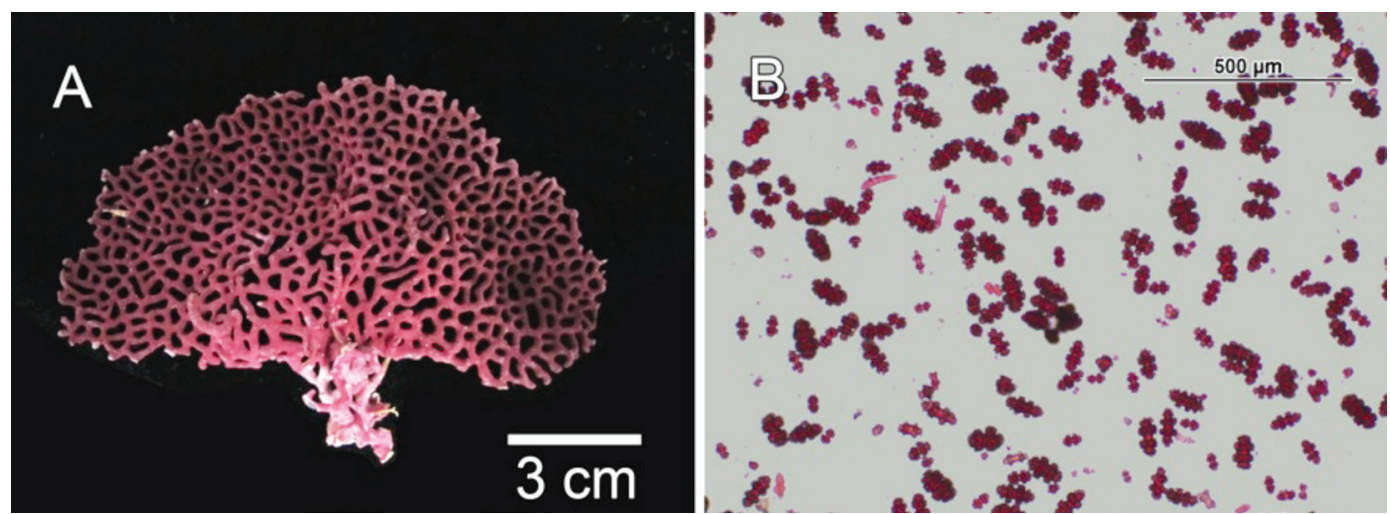

Fig. 21. Pacifigorgia tupperi. (A) Colonia preservada, UCR 904, Islas Murciélago, Costa Rica. (B) Micrografía de escleritas.

Fig. 21. Pacifigorgia tupperi. (A) Preserved colony UCR 904, Islas Murciélago, Costa Rica. (B) Sclerites micrograph. 

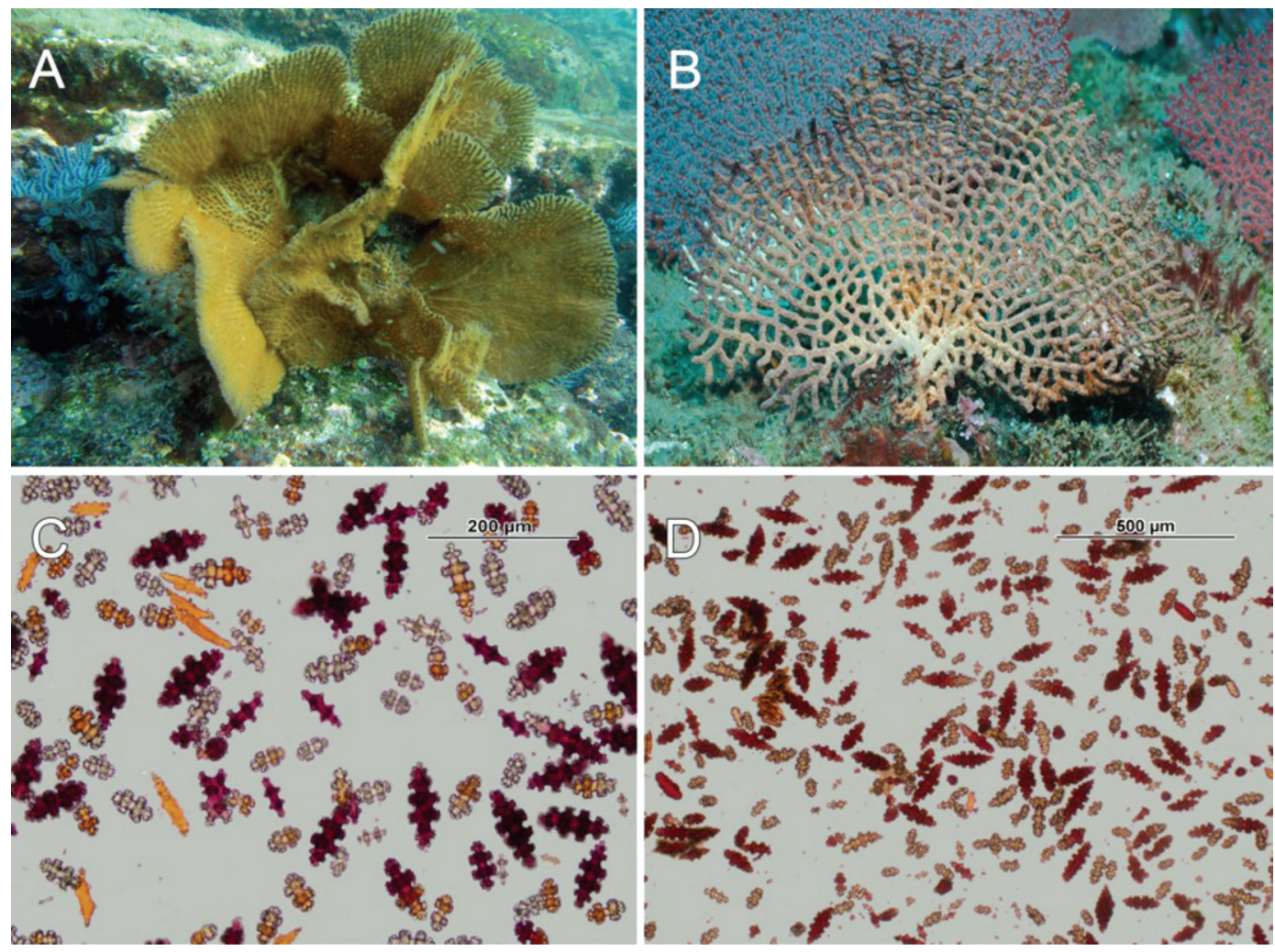

Fig. 22. Pacifigorgia firma. (A) Colonia in situ, Cabo Blanco, Costa Rica, fotografía J. Nivia. (B) Variación interespecífica, Isla Coiba, Panamá, fotografía G. Edgar. (C) Micrografía de escleritas, variación interespecífica, UCR 02-10-2011. (D) Micrografía de escleritas, variación interespecífica UCR 16-3-2013.

Fig. 22. Pacifigorgia firma. (A) In situ colony Cabo Blanco, Costa Rica, photograph J. Nivia. (B) Interspecific variation, Isla Coiba, Panamá, photograph G. Edgar. (C) Sclerites micrograph, interspecific variation, UCR 02-10-2011. (D) Sclerites micrograph, interspecific variation, UCR 16-3-2013.
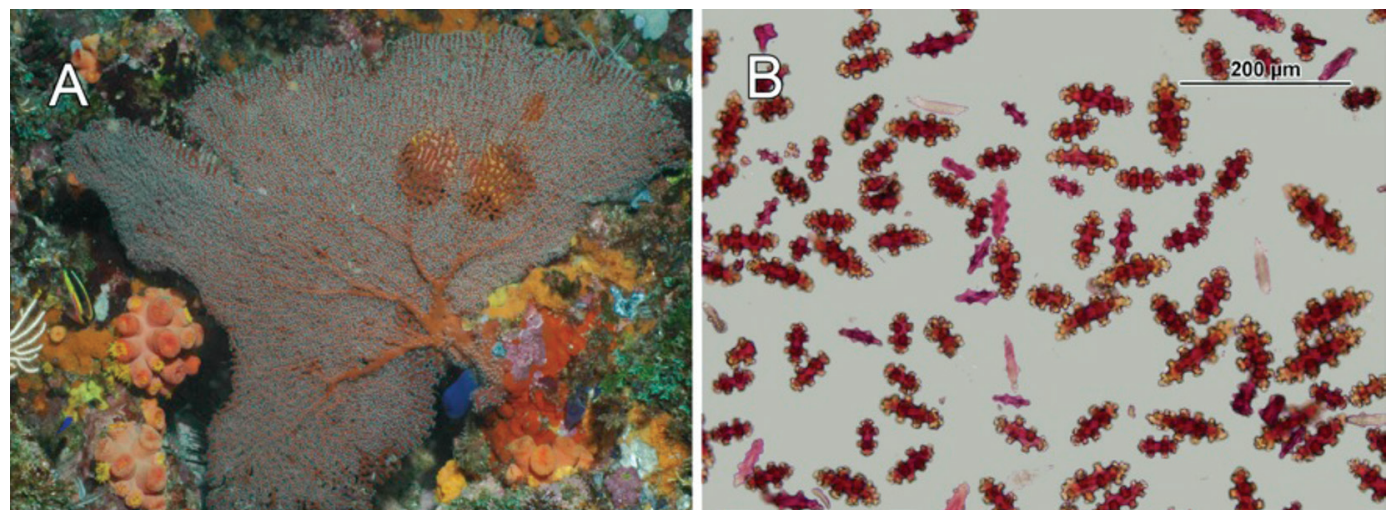

Fig. 23. Pacifigorgia cairnsi. (A) Colonia in situ, Isla Coiba, Panamá, fotografía G. Edgar. (B) Micrografía de escleritas. Fig. 23. Pacifigorgia cairnsi. (A) In situ colony, Coiba Island, Panamá, photograph G. Edgar. (B) Sclerites micrograph. 

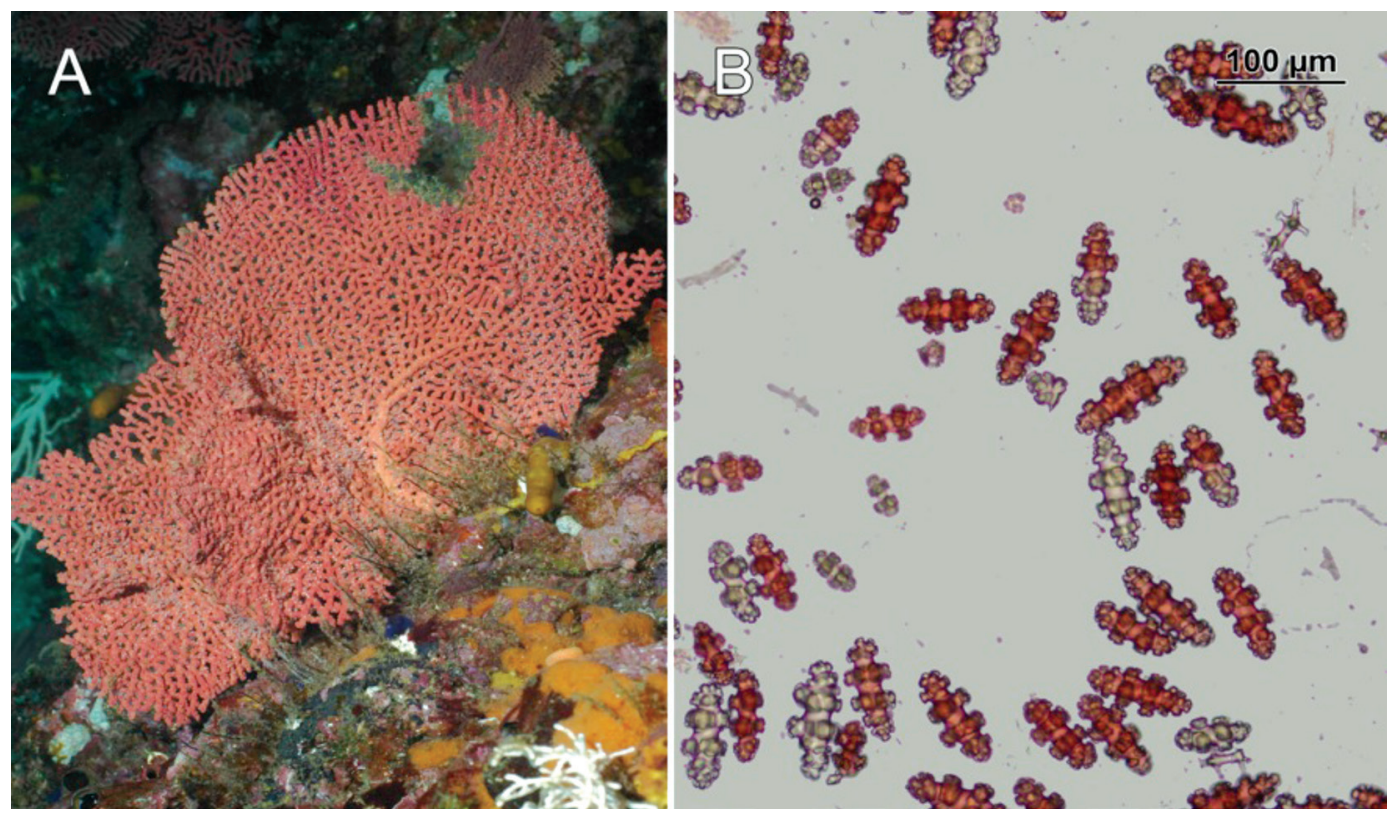

Fig. 24. Pacifigorgia rubicunda. (A) Colonia in situ, Isla Coiba, Panamá, fotografía G. Edgar. (B) Micrografía de escleritas. Fig. 24. Pacifigorgia rubicunda. (A) In situ colony, Coiba Island, Panamá, photograph G. Edgar. (B) Sclerites micrograph, STRI 170.
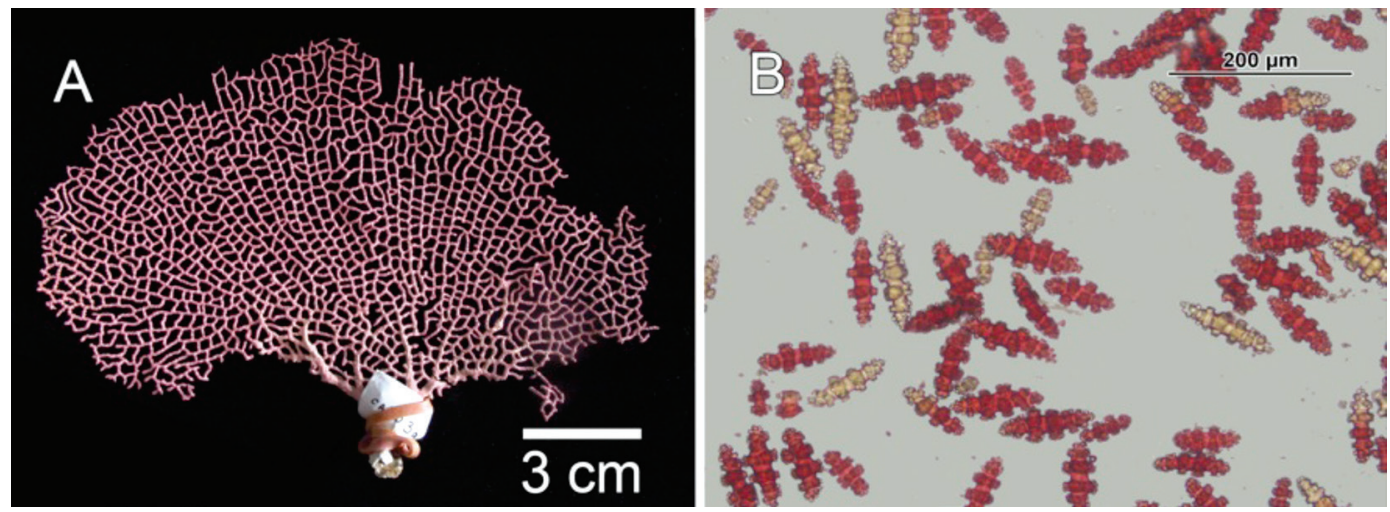

Fig. 25. Pacifigorgia samarensis. (A) Colonia preservada, UCR 959, Isla del Caño, Costa Rica. (B) Micrografía de escleritas, UCR 913.

Fig. 25. Pacifigorgia samarensis. (A) Preserved colony, UCR 959, Caño Island, Costa Rica. (B) Sclerites micrograph, UCR 913.

\section{RESUMEN}

Los octocorales son componentes abundantes y característicos de las aguas someras sobre promontorios rocosos y arrecifes de coral. Se ha informado sobre la riqueza de especies de octocorales de Pacífico de Costa Rica en diversas publicaciones taxonómicos. Sin embargo, no han sido estudiados en cuanto a su ecología, especialmente por los problemas que representa el reconocimiento de las especies, especialmente en el campo y el difícil acceso a las localidades para este tipo de investigación. En 2012 como parte del proyecto Estudios científicos en el área costera del Pacífico Norte, Costa Rica para evaluar el estado de las comunidades coralinas de las aguas someras tuvimos la 
oportunidad de explorar algunos sitios (menos de $35 \mathrm{~m}$ de profundidad) y evaluar la fauna de Octocorallia. Se registró la presencia y la riqueza de especies. Se encontraron cuatro especies en el género Eugorgia, nueve en Leptogorgia y 10 en Pacifigorgia. El objetivo de este trabajo es registrar las especies de octocorales encontradas y facilitar la identificación de esta fauna por medio de la elaboración de una clave para futuros estudios. Las especies se identificaron y caracterizaron morfológicamente, se ilustran con fotografías submarinas, de especímenes preservados y de sus escleritas (estructuras esqueléticas que forman la colonia, que se utilizan en su diferenciación taxonómica).

Palabras clave: biodiversidad, clave para identificación, gorgonias, octocorales, Pacífico oriental, taxonomía.

\section{REFERENCIAS}

Alderslade P. (1984). Subclass Alcyonaria. In: Mather P, Benett I, editors. A coral reef handbook. The Australian Coral Reef Society. p. 45-48.

Bandurraga, M. M., \& Fenical, W. (1985). Isolation of the muricins. Evidence of a chemical adapdation against fouling in the marine octocoral Muricea fruticosa (Gorgonacea). Tetrahedron, 41, 1057-1065.

Baker, J. B., \& Scheuer, P. J. (1994). The punaglandins: 10-Chloroprostanoids from the octocoral Telesto riisei. Journal of Natural Products, 57, 1346-1353.

Bayer, F. M. (1961). The Shallow Water Octocorallia of the West Indian Region. A Manual for Marine Biologists. The Hauge: Martinus Nijhoff. 400 pp.

Bayer, F. M., \& Macintyre, I. G. (2001). The mineral component of the axis and holdfast of some gorgonaceans octocorales (Coelenterata: Anthozoa), with special reference to the family Gorgoniidae. Proceedings of the Biological Society of Washington, 114, 309-345.

Breedy, O. (2001). A new species of Pacifigorgia from the eastern Pacific (Coelenterata: Octocorallia: Gorgoniidae). Bulletin of the Biological Society of Washington, 10, 181-187.

Breedy, O., \& Cortés, J. (2008). Octocorals (Coelenterata: Anthozoa: Octocorallia) of isla del Coco, Costa Rica. Revista de Biología Tropical, 56 (Suppl. 2), 71-77.

Breedy, O., \& Cortés, J. (2011). Morphology and taxonomy of a new species of Leptogorgia (Cnidaria: Octocorallia: Gorgoniidae) in Cocos Island National Park, Pacific Costa Rica. Proceedings of the Biological Society of Washington, 124, 62-69.

Breedy, O., \& Guzman, H.M. (2002). A revision of the genus Pacifigorgia (Coelenterata: Octocorallia: Gorgoniidae). Proceedings of the Biological Society of Washington, 115, 782-839.
Breedy, O., \& Guzman, H. M. (2003a). Octocorals from Costa Rica: The genus Pacifigorgia (Coelenterata: Octocorallia: Gorgoniidae). Zootaxa, 281, 1-60.

Breedy, O., \& Guzmán, H. M. (2003b). A new species of Pacifigorgia (Coelenterata: Octocorallia: Gorgoniidae) from Panamá. Zootaxa, 128, 1-10.

Breedy, O., \& Guzman, H. M. (2004). New species of the gorgoniian genus Pacifigorgia (Coelenterata: Octocorallia: Gorgoniidae) from Pacific Panama. Zootaxa, 541, 1-15.

Breedy, O., \& Guzman, H. M. (2005a). A new species of alcyonacean octocoral from the Galápagos Archipelago. Journal of the Marine Biological Association, U.K., 85, 801-807.

Breedy, O., \& Guzman, H. M. (2005b). A new species of Leptogorgia (Coelenterata: Octocorallia: Gorgoniidae) from the shallow waters of the eastern Pacific. Zootaxa, 899, 1-11.

Breedy, O., \& Guzman, H. M. (2007). A revision of the genus Leptogorgia Milne Edwards \& Haime, 1857 (Coelenterata: Octocorallia: Gorgoniidae) in the eastern Pacific. Zootaxa, 1419, 1-90.

Breedy, O., \& Guzman, H. M. (2008). Leptogorgia ignita, a new shallow-water coral species (Octocorallia: Gorgoniidae) from the tropical eastern Pacific. Journal of the Marine Biological Association, U.K., 88, 893-899.

Breedy, O., \& Guzman, H. M. (2011). A revision of the genus Heterogorgia Verrill, 1868 (Coelenterata: Octocorallia: Plexauridae. Zootaxa, 2995, 27-44.

Breedy, O., \& Guzman, H. M. (2012). A new species of Leptogorgia (Cnidaria: Anthozoa: Octocorallia) from Golfo Dulce, Pacific, Costa Rica. Zootaxa, 3182, 65-68.

Breedy, O., \& Guzman, H. M. (2013). A new species of the genus Eugorgia (Cnidaria: Octocorallia: Gorgoniidae) from mesophotic reefs in the eastern Pacific. Bulletin of Marine Science, 89, 735-743.

Breedy, O, Guzman, H. M. \& Vargas, S. (2009). A revision of the genus Eugorgia Verrill, 1868 (Coelenterata: Octocorallia: Gorgoniidae). Zootaxa, 2151, 1-46.

Breedy, O., Abeytia, R., \& Guzman, H.M. (2012). A new species of Leptogorgia (Cnidaria: Anthozoa: Octocorallia) from the mexican Pacific coast. Bulletin of Marine Science, 88(2), 319-325.

Breedy, O., Williams, G. C., \& Guzman, H. M. (2013) . Two new species of gorgonian octocorals from the Tropical Eastern Pacific Biogeographic Region (Cnidaria, Anthozoa, Gorgoniidae). ZooKeys, 350, 75-90.

Calvo-Shadid, A. \& Breedy-Shadid, O. (2002). Glosario tetralingüe de términos aplicados a la morfología y anatomía de Octocorallia (Coelenterata:Anthozoa). Revista de Filología y Lingüística, 28, 139-153. 
Coll, J. C. (1992). The chemistry and chemical ecology of octocorals (Coelenterata, Anthozoa, Octocorallia). Chemical Reviews, 92, 613-631.

Diaz-Marrero, A. R., Porras, G., Cueto, M., D’Croz, L., Lorenzod, M., San-Martin, A., \& Darias, J. (2009). Leptogorgolide, a biogenetically interesting 1,4-diketo-cembranoid that reinforces the oxidation profile of C-18 as taxonomical marker for octocorals. Tetrahedron, 65, 6029-6033.

Diaz-Marrero, A. R., Porras, G., Aragón, Z., De la Rosa, J. M., Dorta, E., Cueto, M. D’Croz, L., Maté, J., \& Darias, J. (2011). Carijodienone from the octocoral Carijoa multiflora. A Spiropregnane-based steroid. Journal of Natural Products, 74, 292-295.

Fabricius, K. \& Alderslade, P. (2001). Soft Corals and Sea Fans. A Comprehensive Guide to the Tropical Shallow Water Genera of the Central-west Pacific, the Indian Ocean and the Red Sea. Australian Institute of Marine Science. Townsville, Queensland, Australia, 264 pp.

García-Parrado, P. \& Alcolado, P. M. (1996). Catálogo de los octocorales (Cnidaria) de Cuba, con comentarios sobre su taxonomía. Avicennia, 4(5), 41-45.

Gerhardt, D. J. (1990). Fouling and gastropod predation: consequences of grazing from the octocoral. Marine Ecology Progress Series 62, 103-108.

Grasshoff, M. (2001). Coral reef gorgonians of New Caledonia. L'Institut de Recherche pour le Développment, Paris. Collection de Faune et Flore tropicales, 38: $335 \mathrm{p}$.

Gutiérrez, M., Capson, T. L., Guzmán, H. M., González, J., Ortega-Barría, E., \& Quiñoa, E. (2006). Antiplasmodial metabolites isolated from the marine octocoral Muricea austera. Journal of Natural Products, 69, 1379-1383.

Guzman, H. M., \& Breedy, O. (2008). Leptogorgia christiae (Octocorallia: Gorgoniidae) a new shallow gorgonian from Pacific Panama. Journal of the Marine Biological Association, U.K., 88, 719-722.

Guzman, H. M., \& Breedy, O. (2012). Pacifigorgia marviva (Anthozoa: Octocorallia) a new species from Coiba National Park, Pacific Panama. Journal of the Marine Biological Association, U.K., 92, 693-698.

Hickson SJ. (1928). The Gorgonacea of Panama Bay together with a description of one species from the Galápagos Islands and one one of Trinidad. Videnskabelige Meddelelser fra den naturhistoriske Forening $i$ Kjöbenhavn, 85, 325-422.

Jordán-Dahlgren, E. (2002). Gorgonian distribution patterns in coral reef environments of the Gulf of Mexico: evidence of sporadic ecological connectivity? Coral Reefs, 21, 205-215.
Kocurko, J. (1987). Shallow-water Octocorallia and related submarine lithification, San Andres Island, Colombia. Texas Journal of Science, 39, 349-365.

Lasker, H. R. (1985). Prey preferences and browsing pressure of the butterflyfish Chaetodon capistratus on Caribbean gorgonians. Marine Ecology Progress Series 21, 213-220.

Morales, A. (2013) Estudios científicos en el área costera del Pacifico Norte, Costa Rica. Informe Técnico. CIMAR, Universidad de Costa Rica, San Pedro, San José, Costa Rica. 299 pp.

Rodríguez, A. D., \& Ramírez, C. (1994). Further butenolides from the Caribbean octocoral Pterogorgia citrina. Journal of Natural Products, 57, 339-347.

Rodríguez, A. D., Pina, I. C., Soto, J. J., Rojas, D. R., \& Barnes, C. L. (1995). Isolation and structures of the uprolides. I. Thirteen new cytotoxic cembranolides from the Caribbean gorgonian Eunicea mammosa. Canadian Journal of Chemistry, 73, 643-654.

Sammarco, P. W., \& Coll, J. C. (1992) Chemical adaptations in the Octocorallia: evolutionary considerations. Marine Ecology Progress Series, 88, 93-104.

Verrill, A. E. (1868). Critical remarks on the halcyonoid polyps in the Museum of Yale College, with descriptions of new genera. The American Journal of Science and Arts, 45, 411-415.

Verrill, A. E. (1868-70). Notes on Radiata in the Museum of Yale College, Number 6: Review of the corals and polyps of the West Coast of America. Transactions of the Connecticut Academy of Arts and Sciences, 1, 377-558.

Vreeland, H. V., \& Lasker, H. R. (1989). Selective feeding of the polychaete Hermodice carunculata Pallas on Caribbean gorgonians. Journal of Experimental Marine Biology \& Ecology, 129, 265-267.

Williams, G. C., \& Breedy, O. (2004). The Panamic gorgonian genus Pacifigorgia (Octocorallia: Gorgoniidae) in the Galápagos Archipelago, with descriptions of three new species. Proceedings of the California Academy of Sciences, 55, 55-88.

Wirtz, P., \& De Grave, S. (2010). Shrimps (Crustacea, Decapoda, Caridea) associated with gorgonians at the coast of Senegal Arquipelago. Life and Marine Sciences, 27, 69-71.

Wright, A. E., Burres, N. S., \& Schulte, G. K. (1989). Cytotoxic cembranoids from the gorgonian Pseudopterogorgia bipinata. Tetrahedron Letters, 30, 3491-3494.

Zea, S. (1993). Cover of sponges and other sessile organisms in rocky and coral reefs habitats of Santa Marta, Colombian Caribbean Sea. Caribbean Journal Science, 29, 75-88. 